\title{
Unified model beyond grand unification
}

\author{
Juven Wang $\oplus^{*}$ \\ Center of Mathematical Sciences and Applications, Harvard University, Cambridge, Massachusetts 02138, USA
}

(Received 1 January 2021; accepted 12 February 2021; published 27 May 2021)

\begin{abstract}
Strong, electromagnetic, and weak forces were unified in the Standard Model with spontaneous gauge symmetry breaking. These forces were further conjectured to be unified in a simple Lie group gauge interaction in the grand unification. In this work, we propose a theory beyond the Standard Model and grand unification by adding new gapped topological phase sectors consistent with the nonperturbative global anomaly cancellation and cobordism constraints (especially from the baryon minus lepton number $\mathbf{B}-\mathbf{L}$, the electroweak hypercharge $Y$, and the mixed gauge-gravitational anomaly). Gapped topological phase sectors are constructed via symmetry extension, whose low energy contains unitary Lorentz invariant topological quantum field theories (TQFTs): either $(3+1) \mathrm{D}$ noninvertible TQFT (long-range entangled gapped phase), or $(4+1) \mathrm{D}$ invertible or noninvertible TQFT (short-range or long-range entangled gapped phase). Alternatively, there could also be right-handed neutrinos, or gapless unparticle conformal field theories, or their combinations to altogether cancel the mixed gauge-gravitational anomaly. We propose that a new high-energy physics frontier beyond the conventional OD particle physics relies on the new topological force and topological matter including gapped extended objects (gapped 1D line and 2D surface operators or defects, etc., whose open ends carry deconfined fractionalized particle or anyonic string excitations). Physical characterizations of these gapped extended objects require the mathematical theories of cohomology, cobordism, or category. Although weaker than the weak force, topological force is infinite-range or long-range that does not decay in the distance, and mediates between the linked world volume trajectories via fractional or categorical statistical interactions.
\end{abstract}

DOI: 10.1103/PhysRevD.103.105024

\section{INTRODUCTION AND SUMMARY}

Unification is a central theme in theoretical physics. From 1864-1865, Maxwell [1] unified the electricity and magnetism into the electrodynamics theory, where the derived electromagnetic wave manifests the light phenomena. From 1961-1967, Glashow-Salam-Weinberg (GSW) [2-5] made landmark contributions to the electroweak theory of the unified electromagnetic and weak forces between elementary particles, including the prediction of the weak neutral current. The GSW theory together with the strong force is now known as the Standard Model (SM), which is verified to be theoretically and experimentally essential to describe the subatomic high energy physics (HEP). In 1974, GeorgiGlashow [6,7] hypothesized that at a higher energy, the three gauge interactions of the SM would be merged into a single electronuclear force under a simple Lie group gauge theory, known as the grand unification or grand unified theory (GUT).

In this work, follow our previous investigations based on nonperturbative global anomalies and cobordism constraints $[8-10],{ }^{1}$ we propose an ultra unification that a new topological force comes into a theme of unification joining with three known fundamental forces and other hypothetical GUT forces. (See Fig. 1.) More concretely, there is a new gapped

\footnotetext{
jw@ @ cmsa.fas.harvard.edu

${ }^{\mathrm{I}}$ Related global anomalies and cobordism constraints on SM, GUT, and beyond the SM are explored also in [11-14]. Freed gave a generalized cohomology description of the $s u(5)$ GUT anomalies [11]. Garcia-Etxebarria-Montero [12] and Davighi-Gripaios-Lohitsiri [14] used Atiyah-Hirzebruch spectral sequence to compute the classification of global anomalies. The author with Wen [13] and with Wan [8] used Adams spectral sequence [15], Thom-Madsen-Tillmann spectra [16,17], and Freed-Hopkins theorem [18] to obtain the classification of all invertible quantum anomalies. In addition, there in Ref. [8], we can also fully characterize the $d \mathrm{D}$ 't Hooft anomaly [19] of global symmetry $G$ as $(d+1)$ D cobordism invariants, precisely as topological terms of cohomology classes and fermionic topological invariants. The cobordism invariants can be read from the Adams chart in Ref. [8]. Thus we focus on employing Ref. [8] result. By classifying all the invertible quantum anomalies, we must include the following:

(i) All local anomalies (perturbative anomalies): captured by perturbative Feynman diagram loop calculations, classified by the integer $\mathbb{Z}$ classes (the free classes), e.g., Adler-Bell-Jackiw anomalies [20,21], perturbative local gravitational anomalies [22]. Typically the local anomalies are detectable via infinitesimal gauge or diffeomorphism transformations that can be continuously deformed from the identity.

(ii) All global anomalies (nonperturbative anomalies): classified by finite Abelian groups as a product of $\mathbb{Z}_{n}$ (the torsion classes) for some positive integer $n$, e.g., Witten SU(2) [23] and the new SU(2) anomalies [24], global gravitational anomalies [25]. Typically the global anomalies are detectable only via large gauge or diffeomorphism transformations that cannot be continuously deformed from the identity.

More examples of $d \mathrm{D}$ anomalies characterized by $(d+1) \mathrm{D}$ cobordism invariants can be found in [26,27].

Published by the American Physical Society under the terms of the Creative Commons Attribution 4.0 International license. Further distribution of this work must maintain attribution to the author(s) and the published article's title, journal citation, and DOI. Funded by SCOAP ${ }^{3}$.
} 
topological phase sector whose underlying dynamical gauge interactions are the topological forces. In a modern perspective, we should view the SM and GUT all as effective field theories (EFTs) suitable below certain energy scales. Whenever "elementary particles" are mentioned, they only mean to be "elementary field quanta with respect to a given EFT." Likewise, ultra unification should be viewed as an effective field theory which contains SM and GUT but also additional gapped topological phase sectors with low energy Lorentz invariant unitary topological quantum field theories (TQFTs) of Schwarz type (which is the 4D analog of the 3D Chern-SimonsWitten theories [28-30]). Topological force and the gapped topological phase sector here have specific physical and mathematical meanings, which we will clarify in Sec. II F. Before digging into topological phase sector, we should state the assumptions and the logic that lead to the assertion of ultra unification in Sec. II. ${ }^{2}$

\section{LOGIC TO ULTRA UNIFICATION}

\section{A. Assumptions}

Our logic leading to ultra unification starts with the three Assumptions mostly given by nature and broadly confirmed by experiments:

(1) Standard Model gauge group $G_{\mathrm{SM}_{q}}$ : the Standard Model gauge theory has a local Lie algebra $s u(3) \times s u(2) \times u(1)$, but the global structure of Lie group $G_{\mathrm{SM}_{q}}$ has four versions ${ }^{3}$

$$
\begin{gathered}
G_{\mathrm{SM}_{q}} \equiv \frac{\mathrm{SU}(3) \times \mathrm{SU}(2) \times \mathrm{U}(1)}{\mathbb{Z}_{q}}, \\
\text { with } q=1,2,3,6 .
\end{gathered}
$$

\footnotetext{
${ }^{2}$ Conventions: we follow the conventions of Ref. [8-10]. We denote $n \mathrm{D}$ for $n$-dimensional spacetime. We also follow the modern condensed matter terminology on the interacting phases of quantum matter [31,32]. For example, a long-range entangled gapped topological phase whose low energy describes the noninvertible TQFT is known as an intrinsic topological order, which includes examples of fractional quantum Hall states. A short-range entangled gapped topological phase protected by some global symmetry $G$ whose low energy describes the invertible TQFT is known as a symmetryprotected topological state (SPTs), which includes examples of topological insulators and topological superconductors $[33,34]$.

(i) By a noninvertible TQFT, it means that the absolute value partition function $|\mathbf{Z}(M)| \neq 1$ on a generic spacetime manifold $M$ with nontrivial topology (e.g., cycles or homology classes).

(ii) By an invertible TQFT (ITQFT), it means that the absolute value partition function $|\mathbf{Z}(M)|=1$ on any spacetime manifold $M$ with any topology. Thus $\mathbf{Z}(M)$ implies the existence of an inverted phase $\mathbf{Z}^{\prime}(M) \equiv \mathbf{Z}(M)^{-1}$ which defines another ITQFT $\mathbf{Z}^{\prime}(M)$ that can cancel with the original ITQFT $\mathbf{Z}(M)$, as the stacking of two ITQFTs become a trivial vacuum $\mathbf{Z}(M) \cdot \mathbf{Z}^{\prime}(M)=1$ for any $M$.

(iii) By a trivial gapped vacuum with no TQFT or trivial TQFT, it means that the partition function $\mathbf{Z}(M)=1$ on any spacetime manifold $M$ with any topology.

${ }^{3}$ We denote the lower-case $s u, s o, \ldots$ for the Lie algebra and the upper-case $\mathrm{SU}, \mathrm{SO}, \ldots$ for the Lie group.
}

All the quantum numbers of quarks and leptons are compatible with the representations of any version of $q=1,2,3,6$. To confirm which version is used by nature, it requires the experimental tests on extended objects such as 1D line or 2D surface operators (see recent expositions in [35-38]).

SU(5) and Spin(10) gauge group: conventionally, people write the Georgi-Glashow model [6] as the $s u(5)$ GUT and Fritzsch-Minkowski model [7] as the $s o(10)$ GUT because they have the local Lie algebra $s u(5)$ and $s o(10)$, respectively. However, they have the precise global Lie group SU(5) and Spin(10), respectively. Only $q=6$, we are allowed to have the embedding of SM gauge group $G_{\mathrm{SM}_{6}} \equiv$ $\frac{\mathrm{SU}(3) \times \mathrm{SU}(2) \times \mathrm{U}(1)}{\mathbb{Z}_{6}} \subset \mathrm{SU}(5)$ into the $s u(5)$ GUT [see more discussions later in (2.12)].

(2) Observed 15 Weyl fermions per generation by experiments: so far the HEP experiments only confirmed the 15 Weyl fermions per generation of SM. ${ }^{4}$ The experimentalists have not yet confirmed the existence of 16th Weyl fermion for each generation. The 16th Weyl fermion is also known as the right-handed neutrino $\nu_{R}$ or the sterile neutrino. Namely, given the number of generation (or family) $N_{\text {gen }}=3$, we have $15 \times N_{\text {gen }}=45$ Weyl fermions confirmed in the SM. Similarly, we consider $15 \times$ $N_{\text {gen }}=45$ Weyl fermions applicable to the $s u(5)$ GUT. ${ }^{5}$ (The quantum numbers and representations of the elementary particles in SM and GUT can be found in Tables 1 and 2 of [9].) In terms of the quantum numbers of the 15 Weyl fermions per generation of $s u(3) \times s u(2) \times u(1) \mathrm{SM}$ and that of $s u(5)$ GUT, in the left-handed $(L)$ Weyl spinor basis, they are in the representations:

$$
\begin{aligned}
& (\overline{\mathbf{3}}, \mathbf{1}, 1 / 3)_{L} \oplus(\mathbf{1}, \mathbf{2},-1 / 2)_{L} \oplus(\mathbf{3}, \mathbf{2}, 1 / 6)_{L} \\
& \quad \oplus(\overline{\mathbf{3}}, \mathbf{1},-2 / 3)_{L} \oplus(\mathbf{1}, \mathbf{1}, 1)_{L} \text { of } s u(3) \times s u(2) \\
& \quad \times u(1) \sim \overline{\mathbf{5}} \oplus \mathbf{1 0} \text { of } \operatorname{su}(5) .
\end{aligned}
$$

Adding the 16th Weyl fermion (the sterile neutrino) as $(\mathbf{1}, \mathbf{1}, 0)_{L}$ of $s u(3) \times s u(2) \times u(1)$ gives us $\overline{\mathbf{5}} \oplus$ $\mathbf{1 0} \oplus \mathbf{1}$ of $s u(5)$ also $\mathbf{1 6}^{+}$of $s o(10)$ [precisely the 16-dimensional spinor representation of $\operatorname{Spin}(10)]$.

(3) A variant discrete Baryon minus Lepton number (B $-\mathbf{L})$ is preserved at high energy: we hypothesize

\footnotetext{
${ }^{4}$ Here Weyl fermions are spacetime Weyl spinors, which is $\mathbf{2}_{L}$ of $\operatorname{Spin}(1,3)=\operatorname{SL}(2, \mathbb{C})$ with a complex representation in the Lorentz signature. On the other hand, the Weyl spinor is $\mathbf{2}_{L}$ of $\operatorname{Spin}(4)=\mathrm{SU}(2)_{L} \times \mathrm{SU}(2)_{R}$ with a pseudoreal representation in the Euclidean signature.

${ }^{5}$ However, the so(10) GUT requires 16 Weyl fermions per generation due to the fermions sit at the $\mathbf{1 6}$ of the Spin(10). This fact is used to argue the possibility of topological quantum phase transition between the energy scale of the $15 n$ Weyl-fermion $s u(5)$ GUT and 16n Weyl-fermion so(10) GUT in Ref. [10].
} 


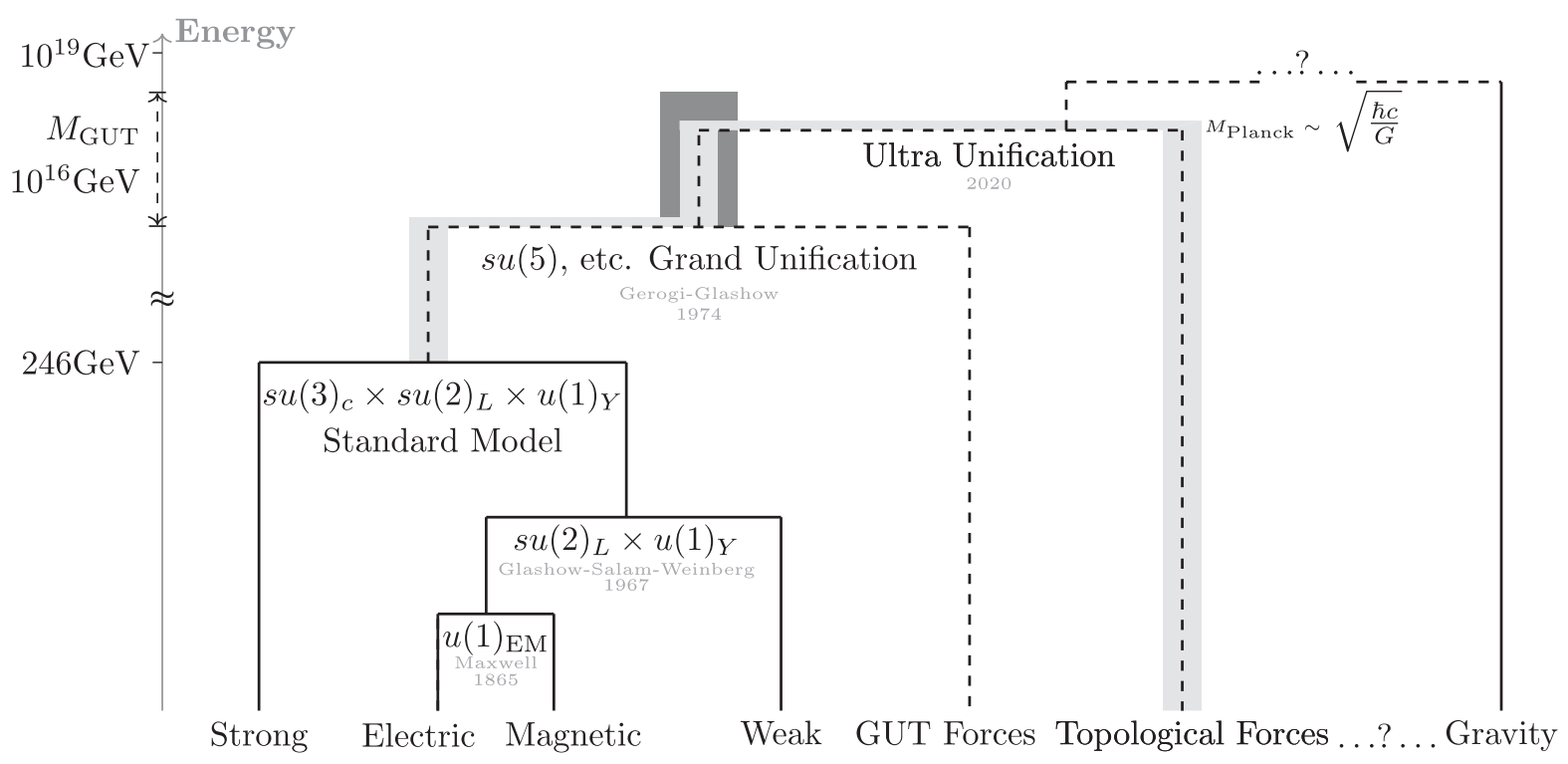

FIG. 1. Unification of forces and interactions in fundamental physics. In fact, the proposed ultra unification can still play a role of unification even if GUT is not favored by nature or verified by experiments. Namely, based on the anomaly and cobordism constraint, we can still propose the SM + gapped topological phase sector + topological force in a consistent way without GUT. The dark gray area

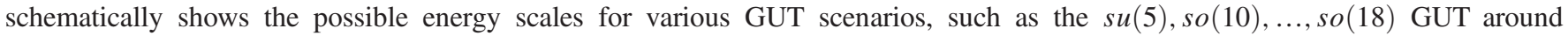
$10^{16} \mathrm{GeV}$. The light gray area schematically suggests that the possible energy gap $\Delta_{\mathrm{TQFT}}$ for topological phase sector can range from as low energy as the SM, to as high energy to somewhere within the GUT scales [e.g., below the so(10) GUT scale]. The dashed lines mean hypothetical unifications that have not yet been confirmed by experiments. Forces are arranged from the strongest to the weakest (horizontally from the left to the right) in the electroweak Higgs vacuum.

a discrete $X$ symmetry, which is a modified version of $(\mathbf{B}-\mathbf{L})$ number up to some electroweak hypercharge $Y$ [39] is preserved (preserved at least at a higher energy): ${ }^{6}$

$$
X \equiv 5(\mathbf{B}-\mathbf{L})-4 Y .
$$

The importance of this discrete symmetry $\mathbb{Z}_{4, X}$ as a mod 4 symmetry of $\mathrm{U}(1)_{X}$ ], in the context of global anomalies for SM and GUT is emphasized by Garcia-Etxebarria-Montero [12].

It is easy to check the following (e.g., see the Tables 1 and 2 of [9]):

(i) All the particles from $\overline{\mathbf{5}}$ of $s u(5)$ GUT has a $\mathrm{U}(1)_{X}$ charge -3 .

(ii) All the particles from $\mathbf{1 0}$ of $s u(5)$ GUT has a $\mathrm{U}(1)_{X}$ charge +1 .

(iii) The singlet right-handed neutrino (if any) is in 1 of $s u(5)$ GUT with a $\mathrm{U}(1)_{X}$ charge +5 .

(iv) All the fermions of SM has a $\mathbb{Z}_{4, X}$ charge +1 .

(v) The electroweak Higgs $\phi$ has a $\mathrm{U}(1)_{X}$ charge -2 , thus a $\mathbb{Z}_{4, X}$ charge +2 .

The $\mathbb{Z}_{4, X}$ also contains the fermion parity $\mathbb{Z}_{2}^{F}$ [whose operator $(-1)^{F}$ gives $(-1)$ to all fermions] as a normal subgroup:

\footnotetext{
${ }^{6}$ Follow [8-10], we choose the convention that the $\mathrm{U}(1)_{\mathrm{EM}}$ electromagnetic charge is $Q_{\mathrm{EM}}=T_{3}+Y$. The $\mathrm{U}(1)_{\mathrm{EM}}$ is the unbroken (not Higgsed) electromagnetic gauge symmetry and $T_{3}=\frac{1}{2}\left(\begin{array}{cc}1 & 0 \\ 0 & -1\end{array}\right)$ is a generator of $\mathrm{SU}(2)_{\text {weak }}$.
}

$$
\mathrm{U}(1)_{X} \supset \mathbb{Z}_{4, X} \supset \mathbb{Z}_{2}^{F}
$$

By looking at these consistent $\mathbb{Z}_{4, X}$ quantum number of SM particles, it is natural to hypothesize the discrete $X$ symmetry plays an important role at a higher energy above the SM energy scale.

In Sec. II B, we review the anomaly and cobordism constraints given in [8-10]. Readers can freely skip the technical discussions on anomalies, and directly go to the final logic step lead to ultra unification in Sec. II C.

\section{B. Anomaly and cobordism constraints}

Based on the three mild and widely accepted assumptions listed in Sec. II A, we then impose the constraints from all invertible quantum anomalies via the cobordism calculation on SM and GUT models. The purpose is to check the consistency of the $15 n$ Weyl fermion SM and GUT models:

Check: Perturbative local and nonperturbative global anomalies classified via cobordism.

The classification of $d \mathrm{D}$ 't Hooft anomalies of global symmetries $G$ is equivalent to the classification of $(d+1) \mathrm{D}$ invertible TQFTs with $G$ symmetry defined on a $G$-structure manifold, ${ }^{7}$ given by the cobordism

\footnotetext{
${ }^{7}$ For the QFT setup, we only require the category of smooth, differentiable, and triangulable manifolds.
} 
group data $\Omega_{G}^{d} \equiv \mathrm{TP}_{d}(G)$ defined in Freed-Hopkins [18]. ${ }^{8}$ The symmetry

$$
\begin{aligned}
G & \equiv\left(\frac{G_{\text {spacetime }} \ltimes G_{\text {internal }}}{N_{\text {shared }}}\right) \\
& \equiv G_{\text {spacetime }} \ltimes_{N_{\text {shared }}} G_{\text {internal }}
\end{aligned}
$$

contains the spacetime symmetry $G_{\text {spacetime }}$ and the internal symmetry $G_{\text {internal }}{ }^{9}$ Our perspective is that

(i) We can treat the spacetime-internal $G$ as a global symmetry, and we view the anomaly associated with $G$ as 't Hooft anomalies [19] of $G$ symmetry.

(ii) Then, we can ask all obstructions to dynamically gauge the $G_{\text {internal }}$ as a gauge group, which gives rise to all the dynamical gauge anomaly cancellation conditions that any consistent gauge theory must obey.

To proceed, we follow the results of $[8,9,40]$, the relevant total spacetime-internal symmetry $G$ for the $\mathrm{SM}_{q}$ is $G=$ $\operatorname{Spin}(d) \times_{\mathbb{Z}_{2}^{F}} \mathbb{Z}_{4, X} \times G_{\mathrm{SM}_{q}}$ with $q=1,2,3,6$, and for the $\operatorname{su}(5)$ GUT is $G=\operatorname{Spin}(d) \times_{\mathbb{Z}_{2}^{F}} \mathbb{Z}_{4, X} \times \mathrm{SU}(5)$. Only the $q=6$ case of $\mathrm{SM}_{6}$ can be embedded into the $s u(5)$ GUT.

\section{Standard models}

Reference [8] considers the classification of $G$ anomalies in $4 \mathrm{D}$ given by the $d=5$ cobordism group $\Omega_{G}^{d} \equiv \mathrm{TP}_{d}(G)$ for all Standard Models of $\mathrm{SM}_{q}$ with an extra discrete $\mathbb{Z}_{4, X}$ symmetry:

\footnotetext{
${ }^{8}$ Let us compare the cobordism group $\Omega_{G}^{d} \equiv \mathrm{TP}_{d}(G)$ defined in Freed-Hopkins [18] and the more familiar bordism group $\Omega_{d}^{G}$. Here the cobordism group $\Omega_{G}^{d} \equiv \mathrm{TP}_{d}(G)$ not only contains $\operatorname{Hom}\left(\Omega_{d}^{G \text {,tors }}, \mathrm{U}(1)\right)$ [the Pontryagin dual of the torsion subgroup (= tors) of the bordism group $\Omega_{d}^{G}$ ], but it also contains the integer $\mathbb{Z}$ classes (the free part) descended from the free part of the bordism group $\Omega_{d+1}^{G \text {,free }}$ of one higher dimension. In other words,

(i) The classification of $(d-1) \mathrm{d}$ nonperturbative global anomalies can be read from the torsion part (the finite subgroup part) of cobordism group $\Omega_{G}^{d \text { tors }} \equiv \operatorname{TP}_{d}^{\text {tors }}(G)$. It can also be read from the torsion part of the bordism group $\Omega_{d}^{G \text { tors }}$ data.

(ii) The classification of $(d-1) \mathrm{d}$ perturbative local anomalies can be read from the free part (the $\mathbb{Z}$ classes) of cobordism group $\Omega_{G}^{d, \text { free }} \equiv \operatorname{TP}_{d}^{\text {free }}(G)$, also from the free part of the bordism group $\Omega_{d+1}^{G \text {,free }}$ data.

In this work, we are concerned the most for $(d-1)=4$ and $d=5$.

${ }^{9}$ The $G_{\text {spacetime }}$ is the spacetime symmetry, such as the spacetime rotational symmetry $\mathrm{SO} \equiv \mathrm{SO}(d)$ or the fermionic graded spacetime rotational spin group symmetry Spin $\equiv$ $\operatorname{Spin}(d)$. The $G_{\text {internal }}$ is the internal symmetry, such as $G_{\text {internal }}$ in the SM as $G_{\mathrm{SM}_{q}} \equiv \frac{\mathrm{SU}(3) \times \mathrm{SU}(2) \times \mathrm{U}(1)}{\mathbb{Z}_{q}}$ with $q=1,2,3,6$. We also have $G_{\text {internal }}=\mathrm{SU}(5)$ in the SU(5) GUT and $G_{\text {internal }}=\operatorname{Spin}(10)$ in the $\operatorname{Spin}(10)$ GUT. The $N_{\text {shared }}$ is the shared common normal subgroup symmetry between $G_{\text {spacetime }}$ and $G_{\text {internal }}$. The "semidirect product $\ltimes$ " extension is due to a group extension from $G_{\text {internal }}$ by $G_{\text {spacetime. }}$. For a trivial extension, the semidirect " $\propto$ " becomes a direct product " $\times$."
}

$$
\begin{aligned}
\mathrm{TP}_{d=5}\left(\operatorname{Spin} \times_{\mathbb{Z}_{2}^{F}} \mathbb{Z}_{4, X} \times G_{\mathrm{SM}_{q}}\right) \\
\quad= \begin{cases}\mathbb{Z}^{5} \times \mathbb{Z}_{2} \times \mathbb{Z}_{4}^{2} \times \mathbb{Z}_{16}, & q=1,3 . \\
\mathbb{Z}^{5} \times \mathbb{Z}_{2}^{2} \times \mathbb{Z}_{4} \times \mathbb{Z}_{16}, & q=2,6 .\end{cases}
\end{aligned}
$$

Here we summarize the anomaly classification for the Standard Model $G_{\mathrm{SM}_{q}} \equiv \frac{\mathrm{SU}(3) \times \mathrm{SU}(2) \times \mathrm{U}(1)_{Y}}{\mathbb{Z}_{q}}$ obtained in $[8,9,40]$. Below we write the $4 \mathrm{D}$ anomalies in terms of the $5 \mathrm{D}$ cobordism invariants or ITQFTs. For perturbative local 4D anomalies, we can also write them customarily as the $6 \mathrm{D}$ anomaly polynomials, and their cubic terms of gauge current couplings in the one-loop triangle Feynman diagram. Here is the list of classifications of anomalies ${ }^{10}$ :

${ }^{10}$ Here we follow the conventions of [8-10]:

(i) We can characterize anomalies via (perturbative) local anomalies or (nonperturbative) global anomalies.

(ii) We can also characterize anomalies via their induced fields: pure gauge anomalies, mixed gauge-gravity anomalies, or gravitational anomalies (those violate the general covariance under coordinate reparametrization; i.e. diffeomorphism).

(iii) The $c_{j}(G)$ is the $j$ th Chern class of the associated vector bundle of the principal $G$ bundle.

(iv) We will use $\mathrm{CS}_{2 n-1}^{V}$ to denote the Chern-Simons (2n-1)form for the Chern class (if $V$ is a complex vector bundle) or the Pontryagin class (if $V$ is a real vector bundle). The relation between the Chern-Simons form and the Chern class is $c_{n}(V)=\mathrm{dCS}_{2 n-1}^{V}$ where the $\mathrm{d}$ is the exterior differential and the $c_{n}(V)$ is regarded as a closed differential form in de Rham cohomology.

(v) The $\mu$ is the 3D Rokhlin invariant. If $\partial M^{4}=M^{3}$, then $\mu\left(M^{3}\right)=\left(\frac{\sigma-\mathrm{F} \cdot \mathrm{F}}{8}\right)\left(M^{4}\right)$, thus $\mu\left(\mathrm{PD}\left(c_{1}(\mathrm{U}(1))\right)\right)$ is related to $\frac{c_{1}(\mathrm{U}(1))(\sigma-\mathrm{F} \cdot \mathrm{F})}{8}$. Here $\cdot$ is the intersection form of $M^{4}$. The F is the characteristic two surface in a four-manifold $M^{4}$; it obeys the condition $\mathrm{F} \cdot x=x \cdot x \bmod 2$ for all $x \in H_{2}\left(M^{4}, \mathbb{Z}\right)$. By the Freedman-Kirby theorem: $\left(\frac{\sigma-\mathrm{F} \cdot \mathrm{F}}{8}\right)\left(M^{4}\right)=\operatorname{Arf}\left(M^{4}, \mathrm{~F}\right) \bmod 2$.

(vi) The PD is defined as the Poincaré dual.

(vii) The $\tilde{\eta}$ is a mod 2 index of $1 \mathrm{D}$ Dirac operator as a cobordism invariant of the bordism group $\Omega_{1}^{\text {Spin }}=\mathbb{Z}_{2}$.

(viii) The $\eta^{\prime}$ is a mod 4 index of 1D Dirac operator as a cobordism invariant of the bordism group $\Omega_{1}^{\operatorname{Spin} \times_{\mathbb{Z}_{2}} \mathbb{Z}_{4}}=\mathbb{Z}_{4}$.

(ix) The Arf invariant [41] is a $2 \mathrm{D}$ cobordism invariant of $\Omega_{2}^{\text {Spin }}=$ $\mathbb{Z}_{2}$. The Arf appears to be the low energy ITQFT of a $(1+1) \mathrm{D}$ Kitaev fermionic chain [42], whose boundary hosts a single $(0+1)$ D real Majorana zero mode on each of open ends.

(x) We use the notation " " to indicate the two sides are equal in that dimension up to a total derivative term.

(xi) Because of the $\mathbb{Z}_{4, X} \supset \mathbb{Z}_{2}^{F}$, we have

$$
0 \rightarrow \mathbb{Z}_{2}^{F} \rightarrow \mathbb{Z}_{4, X} \rightarrow \frac{\mathbb{Z}_{4, X}}{\mathbb{Z}_{2}^{F}}=\mathbb{Z}_{2} \rightarrow 0
$$

We define the cohomology classes of background gauge field $\mathcal{A}_{\mathbb{Z}_{4}} \in H^{1}\left(M, \mathbb{Z}_{4, X}\right)$ as the generator from the cohomology group $H^{1}\left(\mathrm{~B} \mathbb{Z}_{4, X}, \mathbb{Z}_{4}\right)$ of Spin $\times_{\mathbb{Z}_{2}^{F}} \mathbb{Z}_{4, X}$. We also define the background gauge field $\left(\mathcal{A}_{\mathbb{Z}_{2}}\right) \equiv\left(\mathcal{A}_{\mathbb{Z}_{4}}\right) \bmod 2$, where $\mathcal{A}_{\mathbb{Z}_{2}} \in H^{1}\left(M, \mathbb{Z}_{2}\right)$ is the generator from $H^{1}\left(\mathrm{~B}\left(\mathbb{Z}_{4, X} / \mathbb{Z}_{2}^{F}\right), \mathbb{Z}_{2}\right)$ of Spin $\times_{\mathbb{Z}_{2}^{F}} \mathbb{Z}_{4, X}$. 
(1) $\mathrm{U}(1)_{Y}^{3}: 4 \mathrm{D} \mathbb{Z}$ class local pure gauge anomaly from $5 \mathrm{D} \mathrm{CS}{ }_{1}^{\mathrm{U}(1)} c_{1}(\mathrm{U}(1))^{2}$ and $6 \mathrm{D} c_{1}(\mathrm{U}(1))^{3}$.

(2) $\mathrm{U}(1)_{Y}-\mathrm{SU}(2)^{2}: \quad 4 \mathrm{D} \mathbb{Z}$ class local pure gauge anomaly from $5 \mathrm{D} \quad \mathrm{CS}_{1}^{\mathrm{U}(1)} c_{2}(\mathrm{SU}(2))$ and $6 \mathrm{D} c_{1}(\mathrm{U}(1)) c_{2}(\mathrm{SU}(2))$.

(3) $\mathrm{U}(1)_{Y}-\mathrm{SU}(3)^{2}: \quad 4 \mathrm{D} \quad \mathbb{Z}$ class local pure gauge anomaly from $5 \mathrm{D} \mathrm{CS}_{1}^{\mathrm{U}(1)} c_{2}(\mathrm{SU}(3))$ and $6 \mathrm{D} c_{1}(\mathrm{U}(1)) c_{2}(\mathrm{SU}(3))$.

(4) $\mathrm{U}(1)_{Y}$-(gravity $)^{2}: 4 \mathrm{D} \mathbb{Z}$ class local mixed gaugegravity anomaly from $5 \mathrm{D} \mu\left(\mathrm{PD}\left(c_{1}(\mathrm{U}(1))\right)\right)$ and $6 \mathrm{D} \frac{c_{1}(\mathrm{U}(1))(\sigma-\mathrm{F} \cdot \mathrm{F})}{8}$.

(5) $\mathrm{SU}(3)^{3}: 4 \mathrm{D} \mathbb{Z}$ class local pure gauge anomaly from $5 \mathrm{D} \frac{1}{2} \mathrm{CS}_{5}^{\mathrm{SU}(3)}$ and $6 \mathrm{D} \frac{1}{2} c_{3}(\mathrm{SU}(3))$.

(6) Witten $\mathrm{SU}(2)$ anomaly: $4 \mathrm{D} \mathbb{Z}_{2}$ class global mixed gauge-gravity anomaly from $5 \mathrm{D} \quad c_{2}(\mathrm{SU}(2)) \tilde{\eta} \equiv$ $\tilde{\eta}\left(\mathrm{PD}\left(c_{2}(\mathrm{SU}(2))\right)\right) \quad$ and $\quad 6 \mathrm{D} \quad c_{2}(\mathrm{SU}(2)) \operatorname{Arf} \equiv$ $\operatorname{Arf}\left(\operatorname{PD}\left(c_{2}(\mathrm{SU}(2))\right)\right)$. However, the original Witten $\mathbb{Z}_{2}$ global anomaly becomes mutated:

(i) When $q=1$ or 3, Witten anomaly mutated to a $4 \mathrm{D} \quad \mathbb{Z}_{4}$ class global mixed gauge-gravity anomaly, given by a $5 \mathrm{D}$ cobordism invariant $c_{2}(\mathrm{SU}(2)) \eta^{\prime} \equiv \eta^{\prime}\left(\mathrm{PD}\left(c_{2}(\mathrm{SU}(2))\right)\right)$. There is a short exact sequence $0 \rightarrow \mathbb{Z}_{2} \rightarrow \mathbb{Z}_{4} \rightarrow \mathbb{Z}_{2} \rightarrow 0$ where the original Witten anomaly $c_{2}(\mathrm{SU}(2)) \tilde{\eta}$ sits at the $\mathbb{Z}_{2}$ quotient and the $\left(\mathcal{A}_{\mathbb{Z}_{2}}\right) c_{2}(\mathrm{SU}(2))$ sits at the $\mathbb{Z}_{2}$ normal subgroup, while the mutated $c_{2}(\mathrm{SU}(2)) \eta^{\prime}$ sits at the $\mathbb{Z}_{4}$ total group.

(ii) When $q=2$ or 6 , Witten anomaly mutated to become part of $4 \mathrm{D} \mathbb{Z}$ class local mixed gaugegravity anomaly (first explained in [43]), given by a $5 \mathrm{D}$ cobordism invariant $\frac{1}{2} \mathrm{CS}_{1}^{\mathrm{U}(2)} c_{2}(\mathrm{U}(2)) \sim$ $\frac{1}{2} c_{1}(\mathrm{U}(2)) \mathrm{CS}_{3}^{\mathrm{U}(2)}$. There is a short exact sequence $\quad 0 \rightarrow \mathbb{Z}^{2} \rightarrow \mathbb{Z} \rightarrow \mathbb{Z}_{2} \rightarrow 0 \quad$ where the original Witten anomaly $c_{2}(\mathrm{SU}(2)) \tilde{\eta}$ sits at the $\mathbb{Z}_{2}$ quotient and the $\mathrm{CS}_{1}^{\mathrm{U}(1)} c_{2}(\mathrm{SU}(2)) \sim$ $c_{1}(\mathrm{U}(1)) \mathrm{CS}_{3}^{\mathrm{SU}(2)}$ sits at the $\mathbb{Z}$ normal subgroup, while the mutated $\frac{1}{2} \mathrm{CS}_{1}^{\mathrm{U}(2)} c_{2}(\mathrm{U}(2)) \sim$ $\frac{1}{2} c_{1}(\mathrm{U}(2)) \mathrm{CS}_{3}^{\mathrm{U}(2)}$ sits at the $\mathbb{Z}$ total group.

(7) $\left(\mathcal{A}_{\mathbb{Z}_{2}}\right) c_{2}(\mathrm{SU}(2)): 4 \mathrm{D} \mathbb{Z}_{2}$ global gauge anomaly given by a 5D cobordism invariant $\left(\mathcal{A}_{\mathbb{Z}_{4}} \bmod 2\right) c_{2}(\mathrm{SU}(2))$.

(i) When $q=1$ or 3, as explained earlier, it fuses with Witten anomaly to become a $\mathbb{Z}_{4}$ global anomaly.

(ii) When $q=2$ or 6 , this $\mathbb{Z}_{2}$ global anomaly occurs.

(8) $\left(\mathcal{A}_{\mathbb{Z}_{2}}\right) c_{2}(\mathrm{SU}(3)): 4 \mathrm{D} \mathbb{Z}_{2}$ global gauge anomaly.

(i) When $q=1$ or 2 , this is given by a $5 \mathrm{D}$ cobordism invariant $\left(\mathcal{A}_{\mathbb{Z}_{4}} \bmod 2\right) c_{2}(\mathrm{SU}(3))$.

(ii) When $q=3$ or 6 , this is given by a $5 \mathrm{D}$ cobordism invariant $\left(\mathcal{A}_{\mathbb{Z}_{4}} \bmod 2\right) c_{2}(\mathrm{U}(3))$.
(9) $c_{1}(\mathrm{U}(1))^{2} \eta^{\prime}: 4 \mathrm{D} \mathbb{Z}_{4}$ global mixed gauge-gravity anomaly.

(i) When $q=1$, this is given by a $5 \mathrm{D}$ cobordism invariant $c_{1}(\mathrm{U}(1))^{2} \eta^{\prime} \equiv \eta^{\prime}\left(\mathrm{PD}\left(c_{1}(\mathrm{U}(1))^{2}\right)\right)$.

(ii) When $q=2$, this is given by a $5 \mathrm{D}$ cobordism invariant $c_{1}(\mathrm{U}(2))^{2} \eta^{\prime} \equiv \eta^{\prime}\left(\mathrm{PD}\left(c_{1}(\mathrm{U}(2))^{2}\right)\right)$.

(iii) When $q=3$, this is given by a $5 \mathrm{D}$ cobordism invariant $c_{1}(\mathrm{U}(3))^{2} \eta^{\prime} \equiv \eta^{\prime}\left(\mathrm{PD}\left(c_{1}(\mathrm{U}(3))^{2}\right)\right)$.

(iv) When $q=6$, this is given by a $5 \mathrm{D}$ cobordism invariant $c_{1}(\mathrm{U}(2))^{2} \eta^{\prime} \sim c_{1}(\mathrm{U}(3))^{2} \eta^{\prime}$.

(10) $\eta\left(\mathrm{PD}\left(\mathcal{A}_{\mathbb{Z}_{2}}\right)\right): 4 \mathrm{D} \mathbb{Z}_{16}$ global mixed gauge-gravity anomaly is given by a $5 \mathrm{D}$ cobordism invariant $\eta\left(\operatorname{PD}\left(\mathcal{A}_{\mathbb{Z}_{4}} \bmod 2\right)\right)$. The $\eta\left(\operatorname{PD}\left(\mathcal{A}_{\mathbb{Z}_{2}}\right)\right)$ is the value of Atiyah-Patodi-Singer (APS [44]) eta invariant $\eta \in \mathbb{Z}_{16}$ on the Poincare dual (PD) submanifold of $\mathcal{A}_{\mathbb{Z}_{2}}$. It descends from the bordism group calculation of $\Omega_{5}^{\text {Spin } \mathbb{Z}_{2} \mathbb{Z}_{4}}=\Omega_{4}^{\text {Pin }^{+}}=\mathbb{Z}_{16}$. Many previous works had also explored these $5 \mathrm{D} \mathbb{Z}_{16}$ fermionic invariants [45-47]. The relation between $5 \mathrm{D} \eta\left(\operatorname{PD}\left(\mathcal{A}_{\mathbb{Z}_{2}}\right)\right)$ and 4D $\eta$ is given by the Smith homomorphism $[48,49]$.

\section{Georgi-Glashow $\mathrm{su}(5)$ grand unification}

References [8,40] consider the classification of $G$ anomalies in 4D given by the $d=5$ cobordism group $\Omega_{G}^{d} \equiv \mathrm{TP}_{d}(G)$ for the $s u(5)$ GUT with an extra discrete $\mathbb{Z}_{4, X}$ symmetry:

$\mathrm{TP}_{d=5}\left(\operatorname{Spin} \times_{\mathbb{Z}_{2}^{F}} \mathbb{Z}_{4, X} \times \mathrm{SU}(5)\right)=\mathbb{Z} \times \mathbb{Z}_{2} \times \mathbb{Z}_{16}$.

Below we also write down the 4D anomalies in terms of the 5D cobordism invariants or ITQFTs, or the 6D anomaly polynomials:

(1) $\mathrm{SU}(5)^{3}: 4 \mathrm{D} \mathbb{Z}$ class local gauge anomaly. It is given by a $5 \mathrm{D}$ cobordism invariant $\frac{1}{2} \mathrm{CS}_{5}^{\mathrm{SU}(5)}$, or more precisely $\frac{1}{2}\left(\left(\mathcal{A}_{\mathbb{Z}_{2}}\right)^{2} \mathrm{CS}_{3}^{\mathrm{SU}(5)}+\mathrm{CS}_{5}^{\mathrm{SU}(5)}\right)$ including the Spin $\times_{\mathbb{Z}_{2}^{F}} \mathbb{Z}_{4, X}$ contribution.

(2) $\left(\mathcal{A}_{\mathbb{Z}_{2}}\right) c_{2}(\mathrm{SU}(5)): 4 \mathrm{D} \mathbb{Z}_{2}$ class global gauge anomaly. It is given by a $5 \mathrm{D}$ cobordism invariant $\left(\mathcal{A}_{\mathbb{Z}_{4}} \bmod 2\right) c_{2}(\mathrm{SU}(5))$.

(3) $\eta\left(\mathrm{PD}\left(\mathcal{A}_{\mathbb{Z}_{2}}\right)\right): 4 \mathrm{D} \mathbb{Z}_{16}$ global mixed gauge-gravity anomaly is given by a $5 \mathrm{D}$ cobordism invariant $\eta\left(\operatorname{PD}\left(\mathcal{A}_{\mathbb{Z}_{4}} \bmod 2\right)\right)$. This is again the same $\mathbb{Z}_{16}$ global anomaly from $\Omega_{5}^{\text {Spin } \mathbb{Z}_{2} \mathbb{Z}_{4}}=\Omega_{4}^{\text {Pin }^{+}}=\mathbb{Z}_{16}$.

\section{Consequences lead to ultra unification}

References $[9,10]$ checked all the above local and global anomalies enlisted in Sec. II B vanished for the $\mathrm{SM}_{q}$ with $q=1,2,3,6$ and for the $s u(5)$ GUT with 15 Weyl fermions per generation, except the $4 \mathrm{D} \mathbb{Z}_{16}$ class global anomaly [the mixed gauge-gravitational anomaly probed by the discrete $\mathbb{Z}_{4, X}$ symmetry and fermionic spacetime rotational symmetry $\operatorname{Spin}(d)$ background fields] 
may not be completely matched. ${ }^{11}$ The cobordism invariant for any $\nu \in \mathbb{Z}_{16}$ corresponds to a 5D ITQFT partition function

$$
\begin{array}{r}
\mathbf{Z}_{\text {5D-ITQFT }}^{(\nu)} \equiv \exp \left(\left.\frac{2 \pi \mathrm{i}}{16} \cdot \nu \cdot \eta\left(\operatorname{PD}\left(\mathcal{A}_{\mathbb{Z}_{2}}\right)\right)\right|_{M^{5}}\right), \\
\text { with } \eta \equiv \eta_{\text {Pin }^{+}} \in \mathbb{Z}_{16}, \quad \nu \in \mathbb{Z}_{16} .
\end{array}
$$

Given a $G \supseteq \operatorname{Spin} \times_{\mathbb{Z}_{2}^{F}} \mathbb{Z}_{4, X}$ structure, the cohomo$\operatorname{logy}$ class $\mathcal{A}_{\mathbb{Z}_{2}} \in H^{1}\left(M, \mathbb{Z}_{2}\right)$ is the generator from $H^{1}\left(\mathrm{~B}\left(\mathbb{Z}_{4, X} / \mathbb{Z}_{2}^{F}\right), \mathbb{Z}_{2}\right)$. So $\left(\mathcal{A}_{\mathbb{Z}_{2}}\right) \equiv\left(\mathcal{A}_{\mathbb{Z}_{4}}\right) \bmod 2$ is the quotient for the $\mathbb{Z}_{4, X}$ gauge field $\mathcal{A}_{\mathbb{Z}_{4}}$, which is the background field for the symmetry $\mathbb{Z}_{4, X} \subset \mathrm{U}(1)_{X}$. The $\eta\left(\operatorname{PD}\left(\mathcal{A}_{\mathbb{Z}_{2}}\right)\right)$ is the value of $\eta \in \mathbb{Z}_{16}$ on the Poincaré dual (PD) submanifold of the cohomology class $\mathcal{A}_{\mathbb{Z}_{2}}$. This PD takes $\cap \mathcal{A}_{\mathbb{Z}_{2}}$ from 5D to 4D. The APS eta invariant $\eta \equiv \eta_{\mathrm{Pin}^{+}} \in \mathbb{Z}_{16}$ is the cobordism invariant of the bordism group $\Omega_{4}^{\text {Pin }^{+}}=\mathbb{Z}_{16}$. The notation " $\left.\right|_{M^{5}}$ " means the evaluation on this invariant on a 5-manifold $M^{5}$.
We summarize the consequences and implications of this $\mathbb{Z}_{16}$ anomaly non-vanishing for $15 \mathrm{n}$ Weyl fermions in Sec. II C.

(i) Reference [12] used the existence of $\mathbb{Z}_{16}$ global cancellation to verify the conventional lore: the 16 Weyl fermions per generation scenario, by introducing a right-handed neutrino per generation.

(ii) References $[9,10]$ take the $\mathbb{Z}_{16}$ anomaly as a secrete entrance to find hidden new sectors beyond the Standard Model, given the fact the HEP experiments only have detected 15 Weyl fermions per generation thus far.

\section{Consequences}

Given the assumptions in Sec. II A, the $\mathbb{Z}_{16}$ anomaly index for the $\mathrm{SM}_{q}(q=1,2,3,6)$ and the $s u(5)$ GUT both have the $\left(N_{\text {gen }}=3\right)$ and the $(15=-1 \bmod 16)$ per generation. So we have the following anomaly cancellation condition to match the $\mathbb{Z}_{16}$ anomaly:

$$
\left(-\left(N_{\text {gen }}=3\right)+n_{\nu_{e, R}}+n_{\nu_{\mu, R}}+n_{\nu_{\tau, R}}+\text { new hidden sectors }\right)=0 \quad \bmod 16 .
$$

The question is this: how to match the $\mathbb{Z}_{16}$ anomaly? We enlist as many Scenarios ways as possible below.

(1) Standard lore: we can introduce the right-handed neutrino (the 16th Weyl fermion) number $n_{\nu_{R}}=1$ for each generation (so $n_{\nu_{e, R}}=n_{\nu_{\mu, R}}=n_{\nu_{\tau, R}}=1$ for

\footnotetext{
${ }^{11}$ We should briefly compare the perspectives of GarciaEtxebarria-Montero [12], Davighi-Gripaios-Lohitsiri [14], and our previous result $[9,10]$, and those with Wen [13] or with Wan [8]. In terms of the relevancy to the 4D anomalies of SM and $s u(5)$ GUT given the spacetime-internal symmetry $G$

(i) Garcia-Etxebarria-Montero [12] checked $G=\operatorname{Spin} \times_{\mathbb{Z}_{2}} \mathbb{Z}_{4}, \operatorname{Spin} \times \operatorname{SU}(n), \operatorname{Spin} \times \operatorname{Spin}(n)$.

(ii) Wang-Wen [13] checked $G=\frac{\operatorname{Spin} \times \operatorname{Spin}(n)}{\mathbb{Z}_{2}^{F}}, \frac{\operatorname{Spin} \times \operatorname{Spin}(10)}{\mathbb{Z}_{2}^{F}}$, Spin $\times \mathrm{SU}(5)$.

(iii) Davighi-Gripaios-Lohitsiri [14] checked $G=$ Spin $\times$ $G_{\mathrm{SM}_{q}}, \operatorname{Spin} \times \operatorname{Spin}(n)$, and other GUTs.

(iv) Wan-Wang $[8,40] \quad$ checked $G=\operatorname{Spin} \times G_{\mathrm{SM}_{q}}$, $\operatorname{Spin} \times \mathbb{Z}_{2} \mathbb{Z}_{4} \times G_{\mathrm{SM}_{q}}, \quad \operatorname{Spin} \times \operatorname{SU}(5), \quad \operatorname{Spin} \times_{\mathbb{Z}_{2}} \mathbb{Z}_{4} \times \operatorname{SU}(5)$, $\operatorname{Spin} \times \operatorname{Spin}(n), \frac{\operatorname{Spin} \times \operatorname{Spin}(n)}{\mathbb{Z}_{2}^{F}}$, e.g., $n=10,18$, and other GUTs.

Thus, given by the starting assumption in Sec. II A, only WanWang $[8,40]$ contains the complete anomaly classification data for $G=\operatorname{Spin} \times_{\mathbb{Z}_{2}} \mathbb{Z}_{4} \times G_{\mathrm{SM}_{q}}$ and Spin $\times_{\mathbb{Z}_{2}} \mathbb{Z}_{4} \times \mathrm{SU}(5)$ that we need for completing the argument. So we have to employ the results of Ref. [8]. Although Refs. [12,14] checked several global anomalies, they do not exhaust checking all anomalies that we need for $G=\operatorname{Spin} \times_{\mathbb{Z}_{2}} \mathbb{Z}_{4} \times G_{\mathrm{SM}_{q}}$ and Spin $\times_{\mathbb{Z}_{2}} \mathbb{Z}_{4} \times \mathrm{SU}(5)$.

Indeed, specifically for $G=\operatorname{Spin} \times_{\mathbb{Z}_{2}} \mathbb{Z}_{4} \times G_{\mathrm{SM}_{q}}$ and Spin $\times_{\mathbb{Z}_{2}} \mathbb{Z}_{4} \times \mathrm{SU}(5)$, only Refs. $[8,40]$ exhausted the cobordism classifications for all their possible anomalies, and only Refs. $[9,10]$ completed the anomaly cancellation checks.
}

electron, muon, and tau neutrinos). In this case, there is no new hidden sector.

(1a) Massless: $\mathbb{Z}_{4, X}$ can be preserved if fermions are gapless.

(1b) Dirac mass: $\mathbb{Z}_{4, X}$ is alsopreserved by the Yukawa-HiggsDirac Lagrangian, but $\mathbb{Z}_{4, X}$ is spontaneously broken by the Higgs condensate to give a Dirac mass gap.

(1c) Majorana mass: $\mathbb{Z}_{4, X}$ is broken explicitly by Majorana mass term.

(2) Proposals in Refs. [9,10]: Refs. [9,10] proposed other novel ways to match the $\mathbb{Z}_{16}$ anomaly. Consequently, we can introduce new hidden sectors beyond the SM and the $s u(5)$ GUT:

(2a) $\mathbb{Z}_{4, X}$-symmetry-preserving anomalous gapped $4 \mathrm{D}$ TQFT. ${ }^{12}$ We also call the finite energy gap

$$
\Delta_{\mathrm{TQFT}} \equiv E_{\text {excited }}-E_{\text {ground states }}
$$

for the first excitation(s) above the ground state sectors of this $4 \mathrm{D}$ TQFT as topological mass gap. The underlying quantum system has a 4D intrinsic topological order. We name the anomaly index $\nu_{4 \mathrm{D}}$ for this anomalous 4D TQFT.

(2b) $\mathbb{Z}_{4, X}$-symmetry-preserving $5 \mathrm{D}$ ITQFT given by the $5 \mathrm{D}$ cobordism invariant in (2.8). The underlying quantum

\footnotetext{
${ }^{12}$ In the context of 3D boundary and 4D bulk, a novel surface topological order was firstly pointed out by Vishwanath-Senthil in an insightful work [50]. Later on many people follow up on developing the surface topological order constructions (see overviews in [31,32,51]). Reference [9] generalizes this condensed matter idea to find an anomalous symmetric 4D TQFT living on the boundary of 5D fermionic SPTs (2.8).
} 
system has a 5D SPTs with an extra bulk fifth dimension whose 4D boundary can live the 4D Standard Model world. We name the anomaly index $\nu_{5 \mathrm{D}}$ to specify the boundary 4D anomaly of this 5D ITQFT.

(2c) $\mathbb{Z}_{2}^{F}$-symmetry-preseving 5D bulk $\left[\frac{\mathbb{Z}_{4, X}}{\mathbb{Z}_{2}^{F}}\right]$-gauged TQFT and $4 \mathrm{D}$ boundary $\left[\mathbb{Z}_{4, X}\right]$-gauged TQFT. Overall the spacetime rotational symmetry is the fermionic Spin group $\operatorname{Spin}(d)$ graded the bosonic rotation special orthogonal group $\mathrm{SO}(d)$ by the fermion parity $\mathbb{Z}_{2}^{F}$.

(2d) $5 \mathrm{D}$ bulk $\left[\mathbb{Z}_{2}^{F} \times \frac{\mathbb{Z}_{4, X}^{2}}{\mathbb{Z}_{2}^{F}}\right]$-gauged TQFT and $4 \mathrm{D}$ boundary $\left[\mathbb{Z}_{2}^{F} \times \mathbb{Z}_{4, X}\right]$-gauged TQFT. Overall the spacetime rotational symmetry is the bosonic special orthogonal group $\mathrm{SO}(d)$.

(2e) $\mathbb{Z}_{4, X^{-}}$-symmetry-breaking gapped phase (e.g., Ginzburg-Landau paradigm phase or 4D TQFT).

(2f) $\mathbb{Z}_{4, X}$-symmetry-preserving or $\mathbb{Z}_{4, X}$-symmetry-breaking gapless phase, e.g., extra massless theories, free or interacting conformal field theories (CFTs). The interacting CFT with scale invariant gapless energy spectrum is also related to unparticle physics [52] in the high-energy phenomenology community.

Scenarios (2a) and (2b), and their linear combinations, are the root phases, for new hidden gapped topological phase sector. For example, breaking part of the global symmetries in the linear combined (2a) and (2b) would give rise to Scenario (2e). For another example, gauging part of the global symmetries in the linear combined (2a) and (2b) would give rise to other Scenarios:

(i) Gauging $\left[\mathbb{Z}_{4, X}^{\mathbb{F}}\right]$ in $5 \mathrm{D}$ and gauging $\left[\mathbb{Z}_{4, X}\right]$ in $4 \mathrm{D}$ gives rise to scenario $(2 \mathrm{c})$.

(ii) Gauging $\left[\mathbb{Z}_{2}^{F} \times \frac{\mathbb{Z}_{4, X}}{\mathbb{Z}_{2}^{F}}\right]$ in $5 \mathrm{D}$ and gauging $\left[\mathbb{Z}_{2}^{F} \times \mathbb{Z}_{4, X}\right]$ in $4 \mathrm{D}$ gives rise to scenario $(2 \mathrm{~d})$.

The underlying quantum systems in scenarios (2a) and (2b) have the symmetry-enriched topologically ordered state in a condensed matter terminology.

We name the combination of the above anomaly cancellation scenarios, including the standard lore (right-handed neutrinos in Scenario 1) and the new proposals (all enlisted in Scenario 2) beyond the SM and the GUT, as the ultra unification. Although introducing additional conformal field theories [scenario (2f)] to match the anomaly is equally fascinating, we instead mostly focus on introducing the gapped topological phase sector due to high-energy physics phenomenology (HEP-PH) constraints (see a summary in [9]). A central theme of ultra unification suggesting a new HEP frontier is that HEP-PH provides gapped extended objects beyond particle physics. Here are some more comments:

(i) These gapped extended objects (of $1 \mathrm{D}, 2 \mathrm{D}, 3 \mathrm{D}, \ldots$ ) are formulated mathematically in terms of gapped TQFT extended operators (1D line, 2D surface, 3D brane, etc., topological operators), beyond the $0 \mathrm{D}$ particles.

(ii) These extended operators are heavy in the sense that they sit at the energy scale above the TQFT energy gap $\Delta_{\mathrm{TQFT}}$ [so at or above the scale of $E_{\text {excited }}$ in (2.10)]. (iii) These extended operators are heavy in the sense that they have topological mass and they can interact with dynamical gravity. So these gapped extended objects may be the dark matter candidate.

(iv) There are fractionalized anyonic excitations at the open ends of 1D line, 2D surface, 3D brane, etc., topological operators. In other words, the particle 1D worldline is the 1D line topological operator. The anyonic string $2 \mathrm{D}$ worldsheet is the $2 \mathrm{D}$ surface topological operator. ${ }^{13}$

In summary, based on the anomaly cancellation and cobordism constraints, we propose that the SM and Georgi-Glashow SU(5) GUT (with 15 Weyl fermions per generation, and with a discrete baryon minus lepton number $\mathbb{Z}_{4, X}$ preserved) contains a new hidden sector that can be a linear combination of above scenarios $[9,10]$. In particular we can focus on the new hidden sectors given by a 4D TQFT (with the anomaly index $\nu_{4 \mathrm{D}}$ ) and a 5D ITQFT (with the 4D boundary's anomaly index $\nu_{5 \mathrm{D}}$ ), so (2.9) becomes

$\left(-\left(N_{\text {gen }}=3\right)+n_{\nu_{e, R}}+n_{\nu_{\mu, R}}+n_{\nu_{\tau, R}}+\nu_{4 \mathrm{D}}-\nu_{5 \mathrm{D}}\right)=0 \bmod 16$.

\section{Symmetry breaking vs symmetry extension: Dirac or Majorana masses vs topological mass}

The distinctions between Dirac mass, Majorana mass, and topological mass are already explored in Ref. [9]. They represent the scenarios (1b), (1c), and (2a), respectively in Sec. II C. Here we summarize their essences:

(i) Symmetry breaking: Dirac mass and Majorana mass are induced by symmetry breaking - either global symmetry breaking or gauge symmetry breaking, for example via the Anderson-Higgs mechanism or through Yukawa-Higgs term. More precisely, we start from a symmetry group (specifically here an internal symmetry, global or gauged) $G$, and we break $G$ down to an appropriate subgroup $G_{\text {sub }} \subseteq G$ to induce quadratic mass term for matter fields. Mathematically we write an injective homomorphism $t$ :

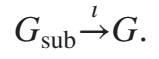

For example, in Anderson-Higgs mechanism, for a Bardeen-Cooper-Schrieffer type $\mathbb{Z}_{2}$-gauged superconductor, we have $G_{\text {sub }}=\mathbb{Z}_{2}$ and $G=\mathrm{U}(1)$ electromagnetic gauge group. For the SM electroweak Higgs mechanism, we have $G_{\text {sub }}=\frac{\mathrm{SU}(3) \times \mathrm{U}(1)_{Y}}{\mathbb{Z}_{\operatorname{gcd}(q, 3)}}$

\footnotetext{
${ }^{13}$ Of course we know that in above $3 \mathrm{D}$ spacetime (such as 4D and 5D that we concern), a OD particle by itself can only have bosonic or fermionic statistics. A OD particle does not have fractional or anyonic statistics [53] in above 3D spacetime. However, 1D worldline and 2D world sheet can be linked in 4D. Triple and quadruple 2D world sheets can be linked in 4D, etc. (see [54-56] and [57-60]). These give rise to "anyonic statistics" to multiexcitations of gapped particles or gapped strings in a 4D spacetime and above.
} 
and $G=G_{\mathrm{SM}_{q}} \equiv \frac{\mathrm{SU}(3) \times \mathrm{SU}(2) \times \mathrm{U}(1)_{\mathrm{EM}}}{\mathbb{Z}_{q}}$ with $q=1,2,3,6$ and the appropriate greatest common divisor (gcd).

(ii) Symmetry extension: topological mass as the energy gap above a TQFT with 't Hooft anomaly (of a spacetime-internal symmetry $G$ ) can be induced by symmetry extension [51]. The symmetry extension mechanism extended the original Hilbert space (with nonperturbative global anomalies) to an enlarged Hilbert space by adding extra degrees of freedom to the original quantum system (see $[61,62]$ for explicit quantum Hamiltonian lattice constructions). The enlarged Hilbert space is meant to trivialize the 't Hooft anomaly in $G$ in an extended $\tilde{G}$. The pullback $r^{*}$ of the map

$$
\tilde{G} \stackrel{r}{\rightarrow} G
$$

can be understood as part of the group extension in an exact sequence [51], while in general this can be generalized as the fibrations of their classifying spaces and higher classifying spaces [63-65]. In many simplified cases, we have a surjective homomorphism $r$ in a short-exact sequence of group extension:

$$
1 \longrightarrow N_{\text {normal }} \longrightarrow \tilde{G} \stackrel{r}{\longrightarrow} G \longrightarrow 1,
$$

where $G=\frac{\tilde{G}}{N_{\text {nomal }}}$ becomes a quotient group of $\tilde{G}$ whose normal subgroup is $N_{\text {normal }}$. The G-anomaly becoming anomaly-free in the extended $\tilde{G}$ requires the essential use of algebraic topology criteria, such as the Lydon-Hochschild-Serre spectral sequence method [51]. We will explain further details in 3.2.1.

\section{E. Gauging a discrete baryon B, lepton $\mathrm{L}$, and electroweak hypercharge $Y$}

We provide some more logical motivations why we should preserve $\mathbb{Z}_{4, X}$ and dynamically gauge $\mathbb{Z}_{4, X}$ with $X \equiv 5(\mathbf{B}-\mathbf{L})-4 Y$, at a higher energy [scenarios (2c) and (2d) in Sec. II C]:

(1) First, as stated before, the $\mathbb{Z}_{4, X}$ is a good global symmetry read from the quantum numbers of SM particles and SM path integral kinematically. It is a global symmetry that has not yet been dynamically gauged in the $G_{\mathrm{SM}_{q}}$ nor in the SU(5) of the $s u(5)$ GUT.

(2) The $\mathbb{Z}_{4, X}=Z(\operatorname{Spin}(10))$ sits at the center subgroup $\mathbb{Z}_{4}$ of the $\operatorname{Spin}(10)$ for the so(10) GUT [12]. Thus the $\mathbb{Z}_{4, X}$ must be dynamically gauged, if the $s o(10)$ GUT is the correct path to unification at a higher energy. It is natural to consider the following group embedding from the GUT to the SM [8-10]:

$$
\begin{aligned}
& \frac{\operatorname{Spin}(d) \times \operatorname{Spin}(10)}{\mathbb{Z}_{2}^{F}} \\
& \supset \operatorname{Spin}(d) \times_{\mathbb{Z}_{2}^{F}} \mathbb{Z}_{4, X} \times \operatorname{SU}(5) \\
& \supset \operatorname{Spin}(d) \times_{\mathbb{Z}_{2}^{F}} \mathbb{Z}_{4, X} \times \frac{\operatorname{SU}(3) \times \operatorname{SU}(2) \times \mathrm{U}(1)}{\mathbb{Z}_{6}} .
\end{aligned}
$$

(i) It is worthwhile mentioning that the $\mathbb{Z}_{16}$ global anomaly (occurred in the cobordism group for the $G=\operatorname{Spin}(d) \times_{\mathbb{Z}_{2}^{F}} \mathbb{Z}_{4, X} \times \mathrm{SU}(5)$ and $\left.\operatorname{Spin}(d) \times_{\mathbb{Z}_{2}^{F}} \mathbb{Z}_{4, X} \times G_{\mathrm{SM}_{q}}\right)$ disappears in the case of $G=\frac{\operatorname{Spin}(d) \times \operatorname{Spin}(10)}{\mathbb{Z}_{2}^{F}}$.

So the $\mathbb{Z}_{4, X}$ can be an anomalous symmetry in the $\mathrm{SM}_{q}$ and the $s u(5)$ GUT, but the $\mathbb{Z}_{4, X}$ is an anomaly-free symmetry in the $s o(10) \mathrm{GUT}^{14}$

(ii) The $15 n$ Weyl fermion $\mathrm{SM}_{q}$ or the $15 n$ Weyl fermion $s u(5)$ GUT alone may have a $\mathbb{Z}_{16}$ global anomaly, while they can become anomaly free at a higher-energy $16 n$ Weyl fermion so(10) GUT [13]. This fact motivates Ref. [10] to propose an analogous concept of topological quantum phase transition happens between two energy scales: (1) above the energy scale of the $\mathrm{SM}_{q}$ or the $s u(5)$ GUT, (2) below the energy scale of the so(10) GUT.

(iii) Global symmetry must be gauged or broken in quantum gravity. If for the above reasons, we ask the $\mathbb{Z}_{4, X}$ symmetry to be preserved, then the $\mathbb{Z}_{4, X}$ must be dynamically gauged at a higher energy for the sake of quantum gravity. ${ }^{15}$

\section{F. Topological phase sector and topological force}

Topological force and the gapped topological phase sector have specific physical and mathematical meanings

\footnotetext{
${ }^{14}$ The anomalous symmetry means a non-on-site symmetry in the condensed matter terminology that cannot be realized acting only locally on a 0 simplex (a point). The anomaly-free symmetry means an on site symmetry in the condensed matter terminology that acts only locally on a 0 simplex (a OD point), which can be easily gauged by coupling to dynamical variables living on 1 simplices (1D line segments).

${ }^{15}$ String theory landscape and swampland develop the similar concepts of the use of cobordism for quantum gravity, see [66] and references therein. This can be understood as the deformation classes of quantum gravity. The deformation classes of quantum field theory is also proposed by Seiberg in [67]. In our context, we propose that the whole quantum system including the low energy SM plus additional hidden sectors, must correspond to the trivial group element 0 class in $\mathrm{TP}_{d=5}\left(\operatorname{Spin} \times_{\mathbb{Z}_{2}^{F}} \mathbb{Z}_{4, X} \times G_{\mathrm{SM}_{q}}\right)$. Similarly, the whole quantum system including the su(5) GUT plus additional hidden sectors, must correspond to the trivial group element 0 class in $\operatorname{TP}_{d=5}\left(\operatorname{Spin} \times_{\mathbb{Z}_{2}^{F}} \mathbb{Z}_{4, X} \times s u(5)\right)$. Anomaly matching: To take a step back, for a usual quantum field theory, one can try to match the index $\nu$ of ' $t$ Hooft anomaly of ultraviolet high energy (UV) with infrared low energy (IR). The index ? is a renormalization group ( $R G)$ flow invariant but possibly can be nonzero. This is the anomaly matching of the index ? between UV and IR theories. Anomaly cancellation: Here in contrast, in our case, we consider the whole quantum system (low energy and high energy) into account, due to our assumption that the $\mathbb{Z}_{4, X}$ is preserved thus gauged at the quantum gravity scale, we must have the system anomaly matched to a trivial group element with the total index $\nu=0$ in the cobordism class (similar to [69]). We may also quote this cancellation as the anomaly matching to zero.
} 
in our context. We should clarify what they are, and then what they are not:

(1) Topological phase sector: in Sec. II C and (2.11), we propose a topological phase sector beyond the Standard Model includes an appropriate linear combination of the following theories (selecting the best scenario to fit into the HEP phenomenology):

(a) 4D long-range entangled gapped topological phase with an energy gap (named the gap $\Delta_{\mathrm{TQFT}}$ ) whose low energy physics is characterized by a $4 \mathrm{D}$ noninvertible topological quantum field theories. This 4D TQFT is a Schwarz-type unitary TQFT (which is the 4D analog of the 3D Chern-Simons-Witten theories [29,30]).

(i) This 4D TQFT has a 't Hooft anomaly [19] of a global symmetry $G$. We named the anomaly index $\nu_{4 \mathrm{D}}$ for this $4 \mathrm{D}$ TQFT.

(ii) Proper mathematical tools to study this $4 \mathrm{D}$ TQFT requires the category or higher category theories.

(b) 5D short-range entangled gapped topological phase with an energy gap whose low energy physics is characterized by a 5D ITQFT. This 5D ITQFT is also a unitary TQFT. But the ITQFT is nontrivial and distinct from a trivial gapped vacuum only in the presence of a global symmetry $G$, see footnote 2 . A $G$-symmetric ITQFT is mathematically given by a $G$-cobordism invariant, classified by an appropriate cobordism group $\Omega_{G}^{d} \equiv \mathrm{TP}_{d}(G)$, defined in the Freed-Hopkins classification of invertible topological phases (TP) [18].

(i) The boundary of this 5D ITQFT has a 4D 't Hooft anomaly of a global symmetry $G$. We named the anomaly index $\nu_{5 \mathrm{D}}$ for this 5D ITQFT.

(ii) Proper mathematical tools to study this 4D TQFT requires characteristic classes, cohomology, and cobordism theories.

(c) 5D long-range entangled gapped topological phase with an energy gap whose low energy physics is characterized by a $5 \mathrm{D}$ TQFT. This is the case when the discrete $X$ symmetry is dynamically gauged, stated in scenarios (2c) and (2d) in Sec. II C.

(2) Topological force: in the context of Sec. IIC, topological force is a discrete gauge force mediated between the linked world volume trajectories (1D worldlines, 2D world sheets from gapped extended operators) via fractional or categorical statistical interactions (see Secs. 5 and 6 of [9]).

(a) Bosonic finite group gauge theory: the conventional discrete gauge theories are bosonic types of finite group gauge theories $[68,69]$. Bosonic types mean that their UV completion only requires a local tensor product Hilbert space of local (gauge-invariant) bosonic operators; the UV completion does not require local (gaugeinvariant) fermionic operators. The underlying TQFT does not require the spin structures and can be defined on non-spin manifolds (such as the oriented SO structures). The TQFTs are known as bosonic or nonspin TQFTs.

(b) Fermionic finite group gauge theory: the discrete gauge theories for our gapped topological phase sectors for the beyond SM hidden sector are fermionic types of finite group gauge theories $[47,64]$. Fermionic types mean that their UV completion must require local (gauge-invariant) fermionic operators. The underlying TQFT requires the additional spin structures defined on spin manifolds. The TQFTs are known as spin TQFTs. In fact the 4D TQFT in Sec. II C requires the Spin $\times_{\mathbb{Z}_{2}^{F}} \mathbb{Z}_{4, X}$ structure and can be defined on the Spin $\times_{\mathbb{Z}_{2}^{F}} \mathbb{Z}_{4, X}$ manifolds (including both spin manifolds and some nonspin manifolds).

We should emphasize that our topological phase sector and topological force are not the kinds of Chern class topological terms which are already summed over in the continuous Lie group gauge theory. Namely, our topological phase sector and topological force are not the following:

(i) The $\theta$ term with or without a dynamical $\theta$ axion [70,71], well known as $\theta F \wedge F$ or $\theta F \tilde{F}$ in the particle physics, is in fact related to the second Chern class $c_{2}\left(V_{G}\right)$ and the square of the first Chern class $c_{1}\left(V_{G}\right)$ of the associated vector bundle of the gauge group $G$ :

$\frac{\theta}{8 \pi^{2}} \operatorname{Tr}(F \wedge F)=\frac{\theta}{2} c_{1}\left(V_{G}\right)^{2}-\theta c_{2}\left(V_{G}\right)$.

In particular, here we consider $G$ as the $\mathrm{U}(N)$ or $\mathrm{SU}(N)$ gauge group, so we can define the Chern characteristic classes associated with complex vector bundles. The $V_{G}$ is the associated vector bundle of the principal $G$ bundle. This $\theta$ term is a topological term, but it is summed over as a weighted factor to define a Yang-Mills gauge theory partition function $[35,72,73]$. This $\theta$ term does not define a quantum system or a quantum phase of matter by itself, distinct from our 4D TQFT (with intrinsic topological order) and 5D ITQFT (with SPTs) as certain unitary quantum phases of matter by themselves.

(ii) The instantons [74,75] or the sphalerons [76] are also not the topological phase sector and topological force in our context. Instantons and sphalerons are again the objects with nontrivial Chern class integrated over the spacetime manifold. Those objects are already defined as part 
of the SM and GUT continuous group gauge theories.

As we will mention in Sec. III A, we can also include (1) the $\theta$ term with or without a dynamical $\theta$ axion, (2) instantons, and (3) sphalerons into the Standard Model and the $s u(5)$ GUT path integral. These objects are already in the old paradigm of the SM and GUT models. These objects do not affect the anomaly cancellation and cobordism constraints (especially the $\mathbb{Z}_{16}$ global anomaly) discussed in Sec. II B. ${ }^{16}$ These objects belong to the Standard Model and the $s u(5)$ GUT path integral (Sec. III A), not to the topological phase sector (TQFT) path integral (Sec. III C), but they all can be included as part of the ultra unification path integral (Sec. III).

\section{ULTRA UNIFICATION PATH INTEGRAL}

In this section, we provide the functional path integral (i.e., partition function) $\mathbf{Z}_{\mathrm{UU}}$ of ultra unification, which includes the standard paradigm of the Standard Model path integral $\mathbf{Z}_{\mathrm{SM}}$ or the Georgi-Glashow $s u(5)$ GUT path integral $\mathbf{Z}_{\mathrm{GUT}}$ in Sec. III A. Then we provide the topological phase sector TQFT path integral $\mathbf{Z}_{\mathrm{TQFT}} \equiv \mathbf{Z}_{5 \mathrm{D} \text {-ITQFT }}$. $\mathbf{Z}_{4 \mathrm{D}-\mathrm{TQFT}}$ in Sec. III C

\section{A. Standard Model and the $s u(5)$ GUT path integral coupled to $X \equiv 5(\mathrm{~B}-\mathrm{L})-4 Y$}

\section{Standard Model path integral coupled to X}

Now we describe the SM path integral in the Minkowski (or Lorentz) signature:

$$
\mathbf{Z}_{\mathrm{SM}}\left[\mathcal{A}_{\mathbb{Z}_{4}}\right] \equiv \int[\mathcal{D} \psi][\mathcal{D} \bar{\psi}][\mathcal{D} A][\mathcal{D} \phi] \ldots \exp \left(\mathrm{i} S_{\mathrm{SM}}\left[\psi, \bar{\psi}, A, \phi, \ldots,\left.\mathcal{A}_{\mathbb{Z}_{4}}\right|_{M^{4}}\right)\right.
$$

The ... depends on the details of which variant versions of SM that we look at (e.g., adding axions or not). In the schematic way, we have the action:

$$
\begin{aligned}
S_{\mathrm{SM}}= & \int_{M^{4}} \sum_{I=1,2,3}\left(+\operatorname{Tr}\left(F_{I} \wedge \star F_{I}\right)-\frac{\theta_{I}}{8 \pi^{2}} g_{I}^{2} \operatorname{Tr}\left(F_{I} \wedge F_{I}\right)\right)+\int_{M^{4}}\left(\bar{\psi}\left(\mathrm{i} D_{A, \mathcal{A}_{\mathbb{Z}_{4}}}\right) \psi\right. \\
& \left.+\left|D_{\mu, A, \mathcal{A}_{\mathbb{Z}_{4}}} \phi\right|^{2}-\mathrm{U}(\phi)-\left(\psi_{L}^{\dagger} \phi \psi_{R}+\text { H.c. }\right)\right) \mathrm{d}^{4} x .
\end{aligned}
$$

But more precisely we really need more details in the Lagrangian $\mathscr{L}$ with Weyl fermions, with $S \equiv \int \mathcal{L} d^{4} x$ :

$$
\begin{aligned}
& \mathcal{L}_{\mathrm{SM}}=\mathcal{L}_{\text {YM }}+\mathcal{L}_{\theta-\text { Chern }}+\mathcal{L}_{\text {Weyl }}+\mathcal{L}_{\text {Higgs }}+\mathcal{L}_{\text {Yukawa-Higgs }} \\
& =\sum_{I=1,2,3}-\frac{1}{4} F_{I, \mu \nu}^{\mathrm{a}} F_{I}^{\mathrm{a} \mu \nu}-\frac{\theta_{I}}{8 \pi^{2}} g_{I}^{2} \epsilon^{\mu \nu \mu^{\prime} \nu^{\prime}} F_{I, \mu \nu}^{\mathrm{a}} F_{I, \mu^{\prime} \nu^{\prime}}^{\mathrm{a}}+\psi_{L}^{\dagger}\left(\mathrm{i}^{\mu} D_{\mu, A, \mathcal{A}_{\mathbb{Z}_{4}}}\right) \psi_{L}+\psi_{R}^{\dagger}\left(\mathrm{i} \sigma^{\mu} D_{\mu, A, \mathcal{A}_{\mathbb{Z}_{4}}}\right) \psi_{R} \\
& +\left|D_{\mu, A, \mathcal{A}_{\mathbb{Z}_{4}}} \phi\right|^{2}-\mathrm{U}(\phi)-\left(\psi_{L}^{\dagger} \phi \psi_{R}+\text { H.c. }\right) \text {. }
\end{aligned}
$$

Here come some remarks:

1 Yang-Mills gauge theory [77] has the action $S_{\mathrm{YM}}=$ $\int \operatorname{Tr}(F \wedge \star F)$ and Lagrangian $\mathcal{L}_{\mathrm{YM}}=-\frac{1}{4} F_{\mu \nu}^{\mathrm{a}} F^{\mathrm{a} \mu \nu}$. The $F$ is the Lie algebra valued field strength curvature two form $F=\mathrm{d} A-\mathrm{i} g A \wedge A$, with its Hodge dual $\star F$, all written in differential forms. In the trace "Tr" we pick up a Lie algebra representation $\mathbf{R}$ whose Lie algebra generators $\mathrm{T}^{a}$ labeled by "a." We have also the subindex $I=1,2,3$ to specify the SM Lie algebra sectors $u(1), s u(2)$, or $s u(3)$.

More precisely $F=\frac{1}{2} F_{\mu \nu}\left(\mathrm{d} x^{\mu} \wedge \mathrm{d} x^{\nu}\right)=$ $\frac{1}{2} F_{\mu \nu}^{a} \mathrm{~T}^{a}\left(\mathrm{~d} x^{\mu} \wedge \mathrm{d} x^{\nu}\right)$, and we define the commutator $\left[\mathrm{T}^{b}, \mathrm{~T}^{c}\right]=\mathrm{i} f^{b c d} \mathrm{~T}^{d}$ with a structure constant $f^{b c d}$,

\footnotetext{
${ }^{16}$ However, those $\theta$ terms in Yang-Mills gauge theory may affect the higher anomalies involving higher generalized global symmetries, see for example [72,73].
}

then $F_{\mu \nu}^{a}=\partial_{\mu} A_{\nu}^{a}-\partial_{\nu} A_{\mu}^{a}+g f^{b c a} A_{\mu}^{b} A_{\nu}^{c}$. Notet that $\operatorname{Tr}\left(\mathrm{T}^{a} \mathrm{~T}^{b}\right)=C(\mathbf{R}) \delta^{a b}$ for some constant of representation $\mathbf{R}$. Here for the fundamental representation $\quad \mathbf{R}$, we take $\operatorname{Tr}\left(\mathrm{T}^{a} \mathrm{~T}^{a}\right)=\frac{1}{2}$. Then we have $\operatorname{Tr}(F \wedge * F)=(-1)^{\mathrm{s}} \frac{1}{2} \operatorname{Tr}\left(F_{\mu \nu} F^{\mu \nu}\right) \mathrm{d}^{4} x=$ $(-1)^{\mathrm{s}}\left(\frac{1}{4}\right) F_{\mu \nu}^{a}\left(F^{a}\right)^{\mu \nu} \mathrm{d}^{4} x$ with the $(-1)^{\mathrm{s}}$ as the sign of the determinant of the spacetime metric.

The $\theta$ term and dynamical $\theta$ axion: we can also introduce the Chern class topological $\theta$ term $S_{\theta \text {-Chern }}=$ $-\int \frac{\theta}{8 \pi^{2}} g^{2} \operatorname{Tr}(F \wedge F)$ and $\mathcal{L}_{\theta \text {-Chern }}=-\frac{\theta}{8 \pi^{2}} g^{2} \epsilon^{\mu \nu \mu^{\prime} \nu^{\prime} \times}$ $F_{\mu \nu}^{\mathrm{a}} F_{\mu^{\prime} \nu^{\prime}}^{\mathrm{a}}$. Given a $\mathrm{U}(N)$ or $\mathrm{SU}(N)$ bundle $V_{G}$ and its field strength $\hat{F}$, the first and second Chern classes are given by $c_{1}\left(V_{G}\right)=\frac{\operatorname{Tr} F}{2 \pi}$ and $c_{2}\left(V_{G}\right)=-\frac{1}{8 \pi^{2}} \operatorname{Tr}(F \wedge F)+$ $\frac{1}{8 \pi^{2}}(\operatorname{Tr} F) \wedge(\operatorname{Tr} F), \quad$ so that $\frac{\theta}{8 \pi^{2}} \operatorname{Tr}(F \wedge F)=$ $\frac{\theta}{2} c_{1}\left(V_{G}\right)^{2}-\theta c_{2}\left(V_{G}\right)$. If a dynamical $\theta$ axion $[70,71]$ is introduced, it requires a summation of the compact $\theta$ in the path integral measure $\int[\mathcal{D} \theta]$. 
There is an overall constant that can be absorbed into the field $A$ and coupling $g$ redefinition. ${ }^{17}$

The path integral $\int[\mathcal{D} A]$ for continuous Lie group gauge field theory [here $\mathrm{U}(1), \mathrm{SU}(N), \mathrm{U}(N)$ for the SM and $s u(5)$ GUT] really means (1) the summation of all inequivalent principal gauge bundles $P_{A}$, and then (2) the summation of all inequivalent gauge connections $\tilde{A}$ (under a given specific principal gauge bundles $P_{A}$ ), where $\tilde{A}$ is a (hopefully globally defined physically) one-form gauge connection. So we physically define:

$$
\int[\mathcal{D} A] \ldots \equiv \sum_{\text {gauge bundle } P_{A}} \int[\mathcal{D} \tilde{A}] \ldots
$$

2 Dirac fermion theory has $S_{\text {Dirac }}=\int \bar{\psi}\left(\mathrm{i} \not D_{A}\right) \psi \mathrm{d}^{4} x$ and $\mathcal{L}_{\text {Dirac }}=\bar{\psi}(\mathrm{i} \not D) \psi$ with the Dirac spinor $\psi$ defined as a section of the spinor bundles. The $\bar{\psi}(\mathrm{i} \not D) \psi$ is an inner product in the complex vector space with the Dirac operator $D_{A}$ as a natural linear operator in the vector space. The path integral $\int[\mathcal{D} \psi][\mathcal{D} \bar{\psi}]$ is (1) the summation of all inequivalent spinor bundles, and then (2) the summation of all inequivalent sections (as spinors) of spinor bundles (under a given specific spinor bundle). We requires spin geometry and spin manifold, in particular we require the Spin $\times_{\mathbb{Z}_{2}} \mathbb{Z}_{4}=\operatorname{Spin} \times_{\mathbb{Z}_{2}^{F}}$ $\mathbb{Z}_{4, X}$ structure.

In fact preferably we present not in the Dirac spinor basis, but we present all of (3.3) in the Weyl spinor basis (below).

3 Weyl fermion theory has $S_{\text {Weyl }}=\int \mathcal{L}_{\text {Weyl }} \mathrm{d}^{4} x=$

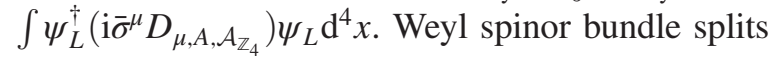
the representation of the Dirac spinor bundle. Weyl spinor again is defined as the section of Weyl spinor bundle. The Weyl spacetime spinor is in $\boldsymbol{2}_{L}$ of $\operatorname{Spin}(1,3)=\operatorname{SL}(2, \mathbb{C})$ with a complex representation in the Lorentz signature, or $\mathbf{2}_{L}$ of $\operatorname{Spin}(4)=$ $\mathrm{SU}(2)_{L} \times \mathrm{SU}(2)_{R}$ with a pseudoreal representation in the Euclidean signature. We also write the analogous right-handed Weyl fermion theory. The $\sigma^{\mu}$ and $\bar{\sigma}^{\mu}$ are the standard spacetime spinor rotational $s u(2)$ Lie algebra generators. We will emphasize and illuminate the meanings of covariant derivative $D_{\mu, A, \mathcal{A}_{\mathbb{Z}_{4}}}$ altogether in remark 5 .

4 Higgs theory has $S_{\text {Higgs }}=\int \mathcal{L}_{\text {Higgs }} \mathrm{d}^{4} x=$ $\int\left(\left|D_{\mu, A, \mathcal{A}_{\mathbb{Z}_{4}}} \phi\right|^{2}-\mathrm{U}(\phi)\right) \mathrm{d}^{4} x$. The Higgs field bundle

\footnotetext{
${ }^{17}$ For example, by redefining $A \rightarrow A^{\prime}=\frac{1}{g} A$ and $F \rightarrow F^{\prime}=\frac{1}{g} F$, then $F^{\prime}=\mathrm{d} A^{\prime}-\mathrm{i} A^{\prime} \wedge A^{\prime} \quad$ and $\quad F_{\mu \nu}^{\prime a}=\partial_{\mu} A_{\nu}^{\prime a}-\partial_{\nu} A_{\mu}^{\prime a}+$ $f^{b c a} A^{\prime b} A_{\mu}^{\prime c}$. Then we can also write $S_{\mathrm{YM}}+S_{\theta \text {-Chern }}=$ $\frac{1}{g^{2}} \int \operatorname{Tr}\left(F^{\prime} \wedge \star F^{\prime}\right)-\int \frac{\theta}{8 \pi^{2}} \operatorname{Tr}\left(F^{\prime} \wedge F^{\prime}\right)$, and $\mathscr{L}_{\mathrm{YM}}+\mathscr{L}_{\theta \text {-Chern }}=$ $-\frac{1}{4 g^{2}} F^{\prime a}{ }_{\mu \nu} F^{\prime a \mu \nu}-\frac{\theta}{8 \pi^{2}} \varepsilon^{\mu \nu \mu^{\prime} \nu^{\prime}} F^{\prime a}{ }_{\mu \nu} F^{\prime a}{ }_{\mu^{\prime} \nu^{\prime}}$.
}

is typically a trivial complex line bundle. ${ }^{18}$ The Higgs scalar field is the section of a field bundle. The electroweak Higgs is in complex value $\mathbb{C}$ and also in 2 of SU(2) gauge field. Again by doing summation $\int[\mathcal{D} \phi]$ we (1) sum over the field bundles, and (2) sum over the section of each field bundle. We illuminate the meanings of covariant derivative $D_{\mu, A, \mathcal{A}_{\mathbb{Z}_{4}}}$ altogether in remark 5 .

5 Covariant derivative operator $D_{\mu, A, \mathcal{A}_{\mathbb{Z}_{4}}}$ in (3.3) is defined as

$$
D_{\mu, A, \mathcal{A}_{\mathbb{Z}_{4}}} \equiv \nabla_{\mu}-\mathrm{i} g q_{\mathbf{R}} A_{\mu}-\mathrm{i} q_{X} \mathcal{A}_{\mathbb{Z}_{4}, \mu} .
$$

Placed on a curved spacetime (with a nondynamical metric, only with background gravity) requires a covariant derivative $\nabla_{\mu}$, and a spin connection for the spinors.

Comments about the term $g q_{\mathbf{R}} A$ in a differential form (e.g., quantum numbers read from Table 1 in [9]):

$$
\begin{aligned}
g q_{\mathbf{R}} A \equiv & \left(q_{e} A_{u(1), \mu}+g_{s u(2)} \sum_{\mathrm{a}=1}^{3} \frac{\varsigma^{\mathrm{a}}}{2} A_{s u(2), \mu}^{\mathrm{a}}\right. \\
& \left.+g_{s u(3)} \sum_{\mathrm{a}=1}^{8} \frac{\tau^{\mathrm{a}}}{2} A_{s u(3), \mu}^{\mathrm{a}}\right) \mathrm{d} x^{\mu} .
\end{aligned}
$$

The $\varsigma^{\mathrm{a}}$ and $\tau^{\mathrm{a}}$ are the rank-2 and rank-3 Lie algebra generator matrix representations for $s u(2)$ and $s u(3)$, respectively. The $D_{\mu, A, \mathcal{A}_{\mathbb{Z}_{4}}}$ acting on $\psi_{L}$ contains the $s u(2)$ gauge field. The $D_{\mu, A, \mathcal{A}_{\mathbb{Z}_{4}}}$ acting on $\psi_{R}$ does not contain the $s u(2)$ gauge field, because the $s u(2)$ weak interaction is a maximally parity violating chiral gauge theory. The $D_{\mu, A, \mathcal{A}_{\mathbb{Z}_{4}}}$ acting only on quarks (both $\psi_{L}$ and $\psi_{R}$ ) contains the $s u(3)$ gauge field. The $D_{\mu, A, \mathcal{A}_{\mathbb{Z}_{4}}}$ acting on $\phi$ contains the $s u(2)$ gauge field.

Here are some comments about the term $q_{X} \mathcal{A}_{\mathbb{Z}_{4}}$ (e.g., quantum numbers read from Tables 1 and 2 in [9]):

(i) The $D_{\mu, A, \mathcal{A}_{\mathbb{Z}_{4}}}$ acts on all left-handed SM Weyl fermion $\psi_{L}$ via its $\mathcal{A}_{\mathbb{Z}_{4}}$ charge $q_{X}=1$.

(ii) The $D_{\mu, A, \mathcal{A}_{\mathbb{Z}_{4}}}$ acts on all right-handed SM Weyl fermion $\psi_{R}$ via its $\mathcal{A}_{\mathbb{Z}_{4}}$ charge $q_{X}=-1$.

(iii) The $D_{\mu, A, \mathcal{A}_{\mathbb{Z}_{4}}}$ acts on the electroweak Higgs $\phi$ via its $\mathcal{A}_{\mathbb{Z}_{4}}$ charge $q_{X}=2$.

The subtle part is that $\mathcal{A}_{\mathbb{Z}_{4}}$ should be treated as a cohomology class, such as a cohomology or cochain gauge field. The $\mathcal{A}_{\mathbb{Z}_{4}} \in H^{1}\left(M, \mathbb{Z}_{4}\right)$ is the generator from $H^{1}\left(\mathrm{~B} \mathbb{Z}_{4, X}, \mathbb{Z}_{4}\right)$. In physics, for the continuum QFT theorists who prefer to think $\mathbb{Z}_{4, X} \subset \mathrm{U}(1)_{X}$ as a continuum

\footnotetext{
${ }^{18}$ In Higgs theory, people in general do not consider nontrivial complex line bundles for Higgs field. But it may be amusing to consider the alternative.
} 
gauge field breaking down to a discrete $\mathbb{Z}_{4, X}$, we can introduce an extra $\mathbb{Z}_{4}$ charge new Higgs field $\varphi$ and its potential $\mathrm{V}(\varphi)$ :

$$
\begin{aligned}
& \psi_{L}^{\dagger}\left(\mathrm{i} \bar{\sigma}^{\mu} D_{\mu, \mathcal{A}_{\mathbb{Z}_{4}}}\right) \psi_{L}+\psi_{R}^{\dagger}\left(\mathrm{i} \sigma^{\mu} D_{\mu, \mathcal{A}_{\mathbb{Z}_{4}}}\right) \psi_{R}+\left|\left(\partial_{\mu}-\mathrm{i} 2 \mathcal{A}_{\mathbb{Z}_{4}, \mu}\right) \phi\right|^{2} \\
& \quad+\left|\left(\partial_{\mu}-\mathrm{i} 4 \mathcal{A}_{\mathbb{Z}_{4}, \mu}\right) \varphi\right|^{2}+\mathrm{V}(\varphi)+\ldots
\end{aligned}
$$

These extra superconductivity-like terms $\phi^{2} \varphi^{\dagger}+\left(\phi^{\dagger}\right)^{2} \varphi$ do not break the $\mathbb{Z}_{4}$. Their $\mathbb{Z}_{4}$ or U(1) transformations are

$\psi_{L} \rightarrow \psi_{L} \mathrm{e}^{\mathrm{i} \frac{2 \pi}{4}}, \quad \psi_{R} \rightarrow \psi_{R} \mathrm{e}^{-\mathrm{i} \frac{2 \pi}{4}}, \quad \phi \rightarrow \phi \mathrm{e}^{\mathrm{i} 2 \frac{2 \pi}{4}}, \quad \varphi \rightarrow \varphi$.

$\psi_{L} \rightarrow \psi_{L} \mathrm{e}^{\mathrm{i} \Theta}, \quad \psi_{R} \rightarrow \psi_{R} \mathrm{e}^{-\mathrm{i} \Theta}, \quad \phi \rightarrow \phi \mathrm{e}^{\mathrm{i} 2 \Theta}, \quad \varphi \rightarrow \varphi \mathrm{e}^{\mathrm{i} 4 \Theta}$.

In the $\langle\varphi\rangle \neq 0$ condensed Higgs phase as a discrete $\mathbb{Z}_{4}$ gauge theory, we can dualize the theory as a level-four BF theory [78]. The formulation starts from adding $\int[D \varphi]$ in the path integral measure, and it ends with a two form $\mathcal{B}$ and one-form gauge field $\mathcal{A}_{\mathbb{Z}_{4}}$

$$
\int[D \mathcal{B}]\left[D \mathcal{A}_{\mathbb{Z}_{4}}\right] \exp \left(\mathrm{i} \frac{4}{2 \pi} \int_{M^{4}} \mathcal{B} \wedge \mathrm{d} \mathcal{A}_{\mathbb{Z}_{4}}+\ldots\right) .
$$

But more precisely, we really should formulate in terms of a cohomology/cochain TQFT and taking care of the Spin $\times_{\mathbb{Z}_{2}^{F}} \mathbb{Z}_{4, X}$ structure, which we will do in Sec. III C (also in Sec. 5 of [9]).

6 Yukawa-Higgs-Dirac term has $S_{\text {Yukawa-Higgs-Dirac }}=$ $\int \mathcal{L}_{\text {Yukawa-Higgs-Dirac }} \mathrm{d}^{4} x=\int\left(\psi_{L}^{\dagger} \phi \psi_{R}+\right.$ H.c. $) \mathrm{d}^{4} x$. In this case, we pair the $\psi_{L}^{\dagger} \mathrm{s} \overline{\mathbf{2}}$ of $\mathrm{SU}(2)$ with the $\phi \mathrm{s}$ 2 of SU(2), and vice versa pair $\psi_{L}$ with $\phi^{\dagger}$ to get an $\mathrm{SU}(2)$ singlet. The right-handed $\psi_{R}$ here is (meant to be) an SU(2) singlet. This Yukawa-Higgs-Dirac term at the kinetic level also preserves the $\mathbb{Z}_{4, X}$, although the Higgs vacuum expectation value breaks the $\mathbb{Z}_{4, X}$ dynamically.

7 Yukawa-Higgs-Majorana term with Weyl fermion: we can add Yukawa-Higgs-Majorana term for Weyl fermions. For example, for the left-handed $\psi_{L}$, we can add a dimension-five operator:

$$
\begin{aligned}
& S_{\text {Yukawa-Higgs-Majorana }} \\
& \quad=\int-\left(\psi_{L}^{\dagger} \phi\left(\phi \mathrm{i} \sigma^{2} \psi_{L}^{*}\right)+\text { H.c. }\right) \mathrm{d}^{4} x \\
& \quad=\int-\left(\psi_{L}^{\dagger} \phi\left(\phi \mathrm{i} \sigma^{2} \psi_{L}^{*}\right)+\left(-\psi_{L}^{\mathrm{T}} \mathrm{i} \sigma^{2} \phi^{*}\right) \phi^{\dagger} \psi_{L}\right) \mathrm{d}^{4} x .
\end{aligned}
$$

Again the $\sigma^{2}$ is from the $\sigma^{\mu}$ of the spacetime spinor rotational $s u(2)$ Lie algebra generators. Renormalizability is not an issue because we are concerned the effective field theory. For the right-handed $\psi_{R}$, we can add a dimension-three operator for some Majorana mass coupling $M$ :

$$
\begin{aligned}
& S_{\text {Yukawa-Higgs-Majorana }} \\
& \quad=\int-M\left(\psi_{R}^{\mathrm{T}}\left(\mathrm{i} \sigma^{2}\right) \psi_{R}+\text { H.c. }\right) \mathrm{d}^{4} x \\
& \quad=\int-M\left(\psi_{R}^{\mathrm{T}}\left(\mathrm{i} \sigma^{2}\right) \psi_{R}+\psi_{R}^{\dagger}\left(-\mathrm{i} \sigma^{2}\right) \psi_{R}^{*}\right) \mathrm{d}^{4} x,
\end{aligned}
$$

which breaks the lepton number conservation. However, in either cases, both Yukawa-Higgs-Majorana terms above break the $\mathbb{Z}_{4, X}$ explicitly. So they are not strongly favored if we pursue the $\mathbb{Z}_{4, X}$-preserving theory at least at higher energy.

We have presented above the Standard Model coupled to a discrete $X$ gauge field in the path integral (3.1), the action (3.2), and the Lagrangian (3.3). Below we can quickly modify a few terms to obtain the $s u(5)$ grand unification coupled to a discrete $X$ gauge field.

\section{The $s u(5)$ grand unification path integral coupled to $X$}

We have the $s u(5)$ GUT path integral coupled to $X$ :

$$
\begin{aligned}
\mathbf{Z}_{\mathrm{GUT}}\left[\mathcal{A}_{\mathbb{Z}_{4}}\right] \equiv & \int[\mathcal{D} \psi][\mathcal{D} \bar{\psi}][\mathcal{D} A][\mathcal{D} \phi] \ldots \\
& \times \exp \left(\mathrm{i} S_{\mathrm{GUT}}\left[\psi, \bar{\psi}, A, \phi, \ldots,\left.\mathcal{A}_{\mathbb{Z}_{4}}\right|_{M^{4}}\right) .\right.
\end{aligned}
$$

We should write all $\overline{\mathbf{5}}$ and $\mathbf{1 0}$ of the SU(5) as the left-handed Weyl fermions $\psi_{L}$, so there are 15 Weyl fermions $\psi_{L}$ per generation. In Sec. II C, we may or may not introduce the right-handed neutrinos here denoted as $\chi_{R}$. In the schematic way, we have the action:

$$
\begin{aligned}
& S_{\mathrm{GUT}}=\int_{M^{4}} \operatorname{Tr}(F \wedge \star F)-\frac{\theta}{8 \pi^{2}} g^{2} \operatorname{Tr}(F \wedge F) \\
& +\int_{M^{4}}\left(\psi_{L}^{\dagger}\left(\mathrm{i} \bar{\sigma}^{\mu} D_{\mu, A, \mathcal{A}_{\mathbb{Z}_{4}}}\right) \psi_{L}+\chi_{R}^{\dagger}\left(\mathrm{i} \sigma^{\mu} D_{\mu, A, \mathcal{A}_{\mathbb{Z}_{4}}}\right) \chi_{R}\right. \\
& +\left|D_{\mu, A, \mathcal{A}_{\mathbb{Z}_{4}}} \phi\right|^{2}-\mathrm{U}(\phi)-\left(\psi_{L}^{\dagger} \phi\left(\sigma^{2} \psi_{L}^{\prime}{ }^{*}\right)\right. \\
& + \text { H.c. })+\ldots) \mathrm{d}^{4} x \text {. }
\end{aligned}
$$

We are left now only with a SU(5) gauge field whose oneform connection is written as

$$
g q_{\mathbf{R}} A=\left(g_{s u(5)} \sum_{\mathrm{a}=1}^{24} \mathrm{~T}^{\mathrm{a}} A_{s u(5), \mu}^{\mathrm{a}}\right) \mathrm{d} x^{\mu} .
$$

We require the $T^{a}$ as the rank-5 and rank-10 Lie algebra generator matrix representations for $s u(5)$ to couple to $\overline{\mathbf{5}}$ and $\mathbf{1 0}$ of $\mathrm{SU}(5)$, respectively. Yukawa-Higgs pairs the appropriate $\psi_{L}$ and $\psi_{L}^{\prime}$ Weyl fermions. We may or may not introduce the Majorana mass terms to $\chi_{R}$ in the ..., while the consequences are already discussed (which break the $\mathbb{Z}_{4, X}$ explicitly) in remark 7 . The discussions about this path integral (3.8) directly follow the above remarks $1-7$, so we should not repeat. 


\section{B. Topological phase sector and TQFT path integral coupled to $X \equiv 5(\mathrm{~B}-\mathrm{L})-4 Y$}

The $15 n$ Weyl fermion SM and $s u(5)$ GUT path integrals, (3.1) and (3.8), are not gauge invariant under the $\mathbb{Z}_{4, X}$ gauge transformation only when the gravitational background is turned on to probe the Spin $\times_{\mathbb{Z}_{2}^{F}} \mathbb{Z}_{4, X^{-}}$-structure.

(i) If the $\mathbb{Z}_{4, X}$ is only coupled to a background gauge field, this only means the system has 't Hooft anomaly under the $\mathbb{Z}_{4, X}$ anomalous symmetry and the spacetime (Spin group) coordinate reparametrization transformations (e.g., the Euclidean rotation or Lorentz boost part of diffeomorphism).

(ii) If the $\mathbb{Z}_{4, X}$ is dynamically gauged and preserved at high energy, then we must append a new sector to make the whole theory well defined.

In any case, following Sec. II C, we now provide a path integral including TQFTs to make the whole theory free from the $\mathbb{Z}_{16}$ global anomaly (2.11):

$$
\left(-\left(N_{\mathrm{gen}}=3\right)+n_{\nu_{e, R}}+n_{\nu_{\mu, R}}+n_{\nu_{\tau, R}}+\nu_{4 \mathrm{D}}-\nu_{5 \mathrm{D}}\right)=0 \bmod 16
$$

(1) The 5D ITQFT partition function is given by (2.8):

$$
\mathbf{Z}_{5 \mathrm{D}-\mathrm{iTQFT}}^{\left(\nu_{\mathrm{S}}\right)}\left[\mathcal{A}_{\mathbb{Z}_{4}}\right] \equiv \exp \left(\left.\frac{2 \pi \mathrm{i}}{16} \cdot \nu_{5 \mathrm{D}} \cdot \eta\left(\operatorname{PD}\left(\mathcal{A}_{\mathbb{Z}_{2}}\right)\right)\right|_{M^{5}}\right), \quad \text { with } \quad \nu \in \mathbb{Z}_{16}, \quad \mathcal{A}_{\mathbb{Z}_{2}} \equiv\left(\mathcal{A}_{\mathbb{Z}_{4}} \bmod 2\right) .
$$

(2) We propose the full gauge invariant path integral, invariant under the mixed gauge-gravity transformation (i.e., gauge-diffeomorphism) of Spin $\times_{\mathbb{Z}_{2}^{F}} \mathbb{Z}_{4, X}$ structure and free from its $\mathbb{Z}_{16}$ global anomaly as follows: The SM version employs (3.1) into

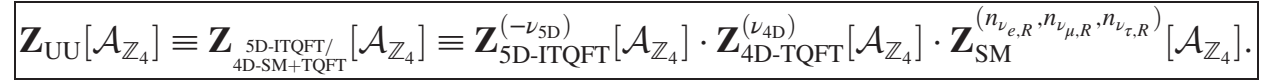

The GUT version employs (3.8) into

$$
\mathbf{Z}_{\mathrm{UU}}\left[\mathcal{A}_{\mathbb{Z}_{4}}\right] \equiv \mathbf{Z}_{\substack{\text { SD-GUTT+TQFT } \\ \text { LD }}}\left[\mathcal{A}_{\mathbb{Z}_{4}}\right] \equiv \mathbf{Z}_{5 \mathrm{D}-\mathrm{ITQFF}}^{\left(-\nu_{\mathrm{D}}\right)}\left[\mathcal{A}_{\mathbb{Z}_{4}}\right] \cdot \mathbf{Z}_{4 \mathrm{D}-\mathrm{TQFT}}^{\left(\nu_{4 \mathrm{D}}\right)}\left[\mathcal{A}_{\mathbb{Z}_{4}}\right] \cdot \mathbf{Z}_{\mathrm{GUT}}^{\left(n_{\nu_{e}, R}, n_{\nu_{\mu, R}}, n_{\nu_{\tau, R}}\right)}\left[\mathcal{A}_{\mathbb{Z}_{4}}\right] .
$$

\section{Symmetry extension $\left[\mathbb{Z}_{2}\right] \rightarrow$ Spin $\times \mathbb{Z}_{4, X} \rightarrow$ Spin $\times_{\mathbb{Z}_{2}^{F}} \mathbb{Z}_{4, X}$ and a $4 D$ fermionic discrete gauge theory}

Below we ask whether we can construct a fully gauge-diffeomorphism invariant 5D-4D coupled partition function preserving the Spin $\times_{\mathbb{Z}_{2}} \mathbb{Z}_{4}$ structure:

$$
\mathbf{Z}_{5 \mathrm{D}-\mathrm{ITQFT}}\left[\mathcal{A}_{\mathbb{Z}_{4}}\right] \cdot \mathbf{Z}_{4 \mathrm{D}-\mathrm{TQFT}}\left[\mathcal{A}_{\mathbb{Z}_{4}}\right] .
$$

Preserving the Spin $\times_{\mathbb{Z}_{2}} \mathbb{Z}_{4}$ structure means that under the spacetime coordinate background transformation (i.e., diffeomorphism) and the $\mathcal{A}_{\mathbb{Z}_{4}}$ background gauge transformation, the 5D-4D coupled partition function is still gaugediffeomorphism invariant.

First, we can rewrite the 5D ITQFT partition function (3.11) on a 5D manifold $M^{5}$ into

$$
\begin{aligned}
\mathbf{Z}_{5 \mathrm{D}-\mathrm{ITQFT}}^{(\nu)}\left[\mathcal{A}_{\mathbb{Z}_{4}}\right] & =\exp \left(\left.\frac{2 \pi \mathrm{i}}{16} \cdot \nu \cdot \eta\left(\operatorname{PD}\left(\mathcal{A}_{\mathbb{Z}_{2}}\right)\right)\right|_{M^{5}}\right) \\
& =\exp \left(\left.\frac{2 \pi \mathrm{i}}{16} \cdot \nu \cdot\left(8 \cdot \frac{p_{1}(T M)}{48}\left(\operatorname{PD}\left(\mathcal{A}_{\mathbb{Z}_{2}}\right)\right)+4 \cdot \operatorname{Arf}\left(\operatorname{PD}\left(\left(\mathcal{A}_{\mathbb{Z}_{2}}\right)^{3}\right)\right)+2 \cdot \tilde{\eta}\left(\operatorname{PD}\left(\left(\mathcal{A}_{\mathbb{Z}_{2}}\right)^{4}\right)\right)+\left(\mathcal{A}_{\mathbb{Z}_{2}}\right)^{5}\right)\right|_{M^{5}}\right) \\
& =\exp \left(\left.\frac{2 \pi \mathrm{i}}{16} \cdot \nu \cdot\left(8 \cdot \frac{\sigma}{16}\left(\operatorname{PD}\left(\mathcal{A}_{\mathbb{Z}_{2}}\right)\right)+4 \cdot \operatorname{Arf}\left(\operatorname{PD}\left(\left(\mathcal{A}_{\mathbb{Z}_{2}}\right)^{3}\right)\right)+2 \cdot \tilde{\eta}\left(\operatorname{PD}\left(\left(\mathcal{A}_{\mathbb{Z}_{2}}\right)^{4}\right)\right)+\left(\mathcal{A}_{\mathbb{Z}_{2}}\right)^{5}\right)\right|_{M^{5}}\right),
\end{aligned}
$$

for a generic $\nu=-N_{\text {generation }} \in \mathbb{Z}_{16}$.

(i) The $p_{1}(T M)$ is the first Pontryagin class of spacetime tangent bundle $T M$ of the manifold $M$. Via the Hirzebruch signature theorem, we have $\frac{1}{3} \int_{\Sigma^{4}} p_{1}(T M)=\sigma\left(\Sigma^{4}\right)=\sigma=\frac{1}{8 \pi^{2}} \int \operatorname{Tr}(R(\omega) \wedge R(\omega))$ on a 4-manifold $\Sigma^{4}$, where $\sigma$ is the signature of $\Sigma^{4}$ while $\omega$ is the 1-connection of tangent bundle and $R(\omega)$ is the Riemann curvature 2-form of $\omega$. So in (3.15), we evaluate the $\frac{1}{3} \int_{\Sigma^{4}} p_{1}(T M)=\sigma$ on the Poincare dual $(\mathrm{PD})$ of $\Sigma^{4}$ manifold of the $\left(\mathcal{A}_{\mathbb{Z}_{2}}\right)$ cohomology class within the $M^{5} .{ }^{19}$

\footnotetext{
${ }^{19}$ Here is a caveat: We know that $\frac{p_{1}(T M)}{48}=\frac{\sigma}{16} \in \mathbb{Z}$ for $4 \mathrm{~d}$ spin manifolds which makes (3.15) computable. But we leave the precise analogous revised expression of $\frac{p_{1}(T M)}{48}=\frac{\sigma}{16}$ on unoriented manifolds (such as Pin ${ }^{+}$manifolds) as a mod 2 class [79-81] in the parallel work [82].
} 
(ii) The $\tilde{\eta}$ is a mod 2 index of 1D Dirac operator as a cobordism invariant of the bordism group $\Omega_{1}^{\text {Spin }}=\mathbb{Z}_{2}$. The $1 \mathrm{~d}$ manifold generator of $\tilde{\eta}$ is a circle $S^{1}$ with a periodic boundary condition (i.e., Ramond) for the fermion.

(iii) The Arf invariant [41] is a mod 2 cobordism invariant of the bordism group $\Omega_{2}^{\text {Spin }}=\mathbb{Z}_{2}$, whose realization is the $(1+1) \mathrm{D}$ Kitaev fermionic chain [42] whose open end hosts a $0+1 \mathrm{~d}$ Majorana zero mode.

(iv) The $\left(\mathcal{A}_{\mathbb{Z}_{2}}\right)^{5}$ is a mod 2 class purely bosonic topological invariant, which corresponds to a $5 \mathrm{D}$ bosonic SPT phase given by the group cohomology class data $H^{5}\left(\mathrm{~B} \mathbb{Z}_{2}, \mathrm{U}(1)\right)=\mathbb{Z}_{2}$, which is also one of the $\mathbb{Z}_{2}$ generators in $\Omega_{5}^{\mathrm{SO}}\left(\mathrm{B} \mathbb{Z}_{2}\right)$.

(a) When $\nu$ is odd, such as $\nu=1,3,5,7, \ldots \in \mathbb{Z}_{16}$, Ref. [46] suggested that the symmetry-extension method [51] cannot construct a symmetry-gapped TQFT. Furthermore, Cordova-Ohmori [83] proves that a symmetry-preserving gapped TQFT phase is impossible for this odd $\nu \in \mathbb{Z}_{16}$ anomaly from $\Omega_{5}^{\operatorname{Spin} \times \mathbb{Z}_{2} \mathbb{Z}_{4}}=\mathbb{Z}_{16}$. The general statement in [83] is that given an anomaly index $\nu \in \Omega_{5}^{\operatorname{Spin} \times \mathbb{Z}_{2} \mathbb{Z}_{4}}=$ $\mathbb{Z}_{16}$, we can at most construct a fully symmetric gapped TQFT if and only if $4 \mid 2 \nu$. Namely, 4 has to be a divisor of $2 \nu$. Apparently, the $4 \mid 2 \nu$ is true only when $\nu$ is even.

Since $\nu=-N_{\text {generation, }}$, the case of $\nu=1$ (for a single generation) and $\nu=3$ (for three generations) are particularly important for the high energy physics phenomenology. This means that we are not able to directly construct any 4D symmetric gapped TQFT that explicitly matches the same $\mathbb{Z}_{16}$ anomaly for one right-handed neutrino $(\nu=1)$ or three right-handed neutrinos $(\nu=3)$.

(b) When $\nu$ is even, such as $\nu=2,4,6,8, \ldots \in \mathbb{Z}_{16}$, Refs. [38,46,61,82] suggested that the symmetry-extension method [51] can trivialize the 't Hooft anomaly. Furthermore, Cordova-Ohmori [83] shows that there is no obstruction to construct a symmetry-preserving gapped TQFT phase for any even $\nu_{\text {even }} \in \mathbb{Z}_{16}$. We can verify the claim by rewriting (3.15) in terms of the $\left(\frac{\nu_{\text {even }}}{2}\right) \in \mathbb{Z}_{8}$ index:

$$
\begin{aligned}
\mathbf{Z}_{\text {5D-ITQFT }}^{\left(\nu_{\text {even }}=2\right)} & =\exp \left(\left.\frac{2 \pi \mathrm{i}}{16} \cdot \nu_{\text {even }} \cdot \eta\left(\operatorname{PD}\left(\mathcal{A}_{\mathbb{Z}_{2}}\right)\right)\right|_{M^{5}}\right) \\
& =\exp \left(\left.\frac{2 \pi \mathrm{i}}{8} \cdot\left(\frac{\nu_{\text {even }}}{2}\right) \cdot\left(\operatorname{ABK}\left(\operatorname{PD}\left(\left(\mathcal{A}_{\mathbb{Z}_{2}}\right)^{3}\right)\right)\right)\right|_{M^{5}}\right) \\
& =\exp \left(\left.\frac{2 \pi \mathrm{i}}{8} \cdot\left(\frac{\nu_{\text {even }}}{2}\right) \cdot\left(4 \cdot \operatorname{Arf}\left(\operatorname{PD}\left(\left(\mathcal{A}_{\mathbb{Z}_{2}}\right)^{3}\right)\right)+2 \cdot \tilde{\eta}\left(\operatorname{PD}\left(\left(\mathcal{A}_{\mathbb{Z}_{2}}\right)^{4}\right)\right)+\left(\mathcal{A}_{\mathbb{Z}_{2}}\right)^{5}\right)\right|_{M^{5}}\right),
\end{aligned}
$$

with a 2D Arf-Brown-Kervaire (ABK) invariant. which is also known as the Pin $^{-}$-structure $\mathbb{Z}_{8}$ class of iTQFT of the $1+1 \mathrm{~d}$ Fidkowski-Kitaev fermionic chain $[84,85]$ with a time reversal $T^{2}=+1$ symmetry. Notice that (3.16) can become trivialized if we can trivialize the $\left(\mathcal{A}_{\mathbb{Z}_{2}}\right)^{3}$ factor. In fact, the $\left(\mathcal{A}_{\mathbb{Z}_{2}}\right)^{3}$ can be trivialized by the symmetry extension [51], written in terms of the group extension of a short exact sequence:

$$
0 \rightarrow \mathbb{Z}_{2} \rightarrow \mathbb{Z}_{4, X} \rightarrow \frac{\mathbb{Z}_{4, X}}{\mathbb{Z}_{2}^{F}} \rightarrow 0
$$

Namely, the two-cocycle topological term $\left(\mathcal{A}_{\mathbb{Z}_{2}}\right)^{3}$ in $H^{2}\left(\mathrm{~B}\left(\frac{\mathbb{Z}_{4, X}}{\mathbb{Z}_{2}^{F}}\right), \mathrm{U}(1)\right)$ becomes a coboundary once we lifting the $\frac{\mathbb{Z}_{4, X}}{\mathbb{Z}_{2}^{F}}$ gauge field $\mathcal{A}_{\mathbb{Z}_{2}}$ to a $\mathbb{Z}_{4, X}$ gauge field in $H^{3}\left(\mathrm{~B} \mathbb{Z}_{4, X}, \mathrm{U}(1)\right)$. So this suggests that the following symmetry extension for the spacetime-internal symmetry, written in terms of the group extension of a short exact sequence, ${ }^{20}$

\footnotetext{
${ }^{20}$ See more discussion in Sec. 5 of [9] and in [82].
}

$$
1 \rightarrow\left[\mathbb{Z}_{2}\right] \rightarrow \operatorname{Spin} \times \mathbb{Z}_{4, X} \rightarrow \operatorname{Spin} \times_{\mathbb{Z}_{2}^{F}} \mathbb{Z}_{4, X} \rightarrow 1,
$$

can fully trivialize any even $\nu_{\text {even }} \in \mathbb{Z}_{16}$ cobordism invariant given in (3.16). The $\left[\mathbb{Z}_{2}\right]$ means that we can gauge the anomaly-free normal subgroup $\left[\mathbb{Z}_{2}\right]$ in the total group Spin $\times \mathbb{Z}_{4, X}$. This symmetry extension (3.17) also means that a $4 \mathrm{D}\left[\mathbb{Z}_{2}\right]$ gauge theory preserves the Spin $\times_{\mathbb{Z}_{2}^{F}} \mathbb{Z}_{4, X}$ symmetry while also saturates the even $\nu_{\text {even }} \in \mathbb{Z}_{16}$ anomaly. This $4 \mathrm{D}$ $\left[\mathbb{Z}_{2}\right]$ gauge theory is the anomalous symmetric gapped noninvertible TQFT (with 't Hooft anomaly of Spin $\times_{\mathbb{Z}_{2}^{F}} \mathbb{Z}_{4, X}$ symmetry) desired in scenario (2a).

Since a symmetric anomalous 4D TQFT only exists with even $\nu_{\text {even }} \in \mathbb{Z}_{16}$, below we formulate the path integral $\mathbf{Z}_{4 \mathrm{D}-\mathrm{TQFT}}^{\left(\nu_{4 \mathrm{D}}=2\right)}\left[\mathcal{A}_{\mathbb{Z}_{4}}\right]$ of the root phase $\nu_{4 \mathrm{D}}=2$. We generalize the boundary TQFT construction in the Sec. 8 of [47]. With $\nu_{\text {even }}=2 \in \mathbb{Z}_{16}$, we have (3.14) with the input of 5D bulk ITQFT (3.16), then we can explicitly 
construct the partition function on a 5D manifold $M^{5}$ with a $4 \mathrm{D}$ boundary $M^{4} \equiv \partial M^{5} \mathrm{as}^{21}$

$$
\begin{aligned}
& \mathbf{Z}_{5 \text { D-ITQFT }}\left[\mathcal{A}_{\mathbb{Z}_{4}}\right] \cdot \mathbf{Z}_{4 \mathrm{D}-\mathrm{TQFT}}\left[\mathcal{A}_{\mathbb{Z}_{4}}\right] \\
& =\sum_{c \in \partial^{\prime-1}\left(\partial\left[\operatorname{PD}\left(\mathcal{A}^{3}\right)\right]\right)} \mathrm{e}^{\frac{2 \pi i}{8} \mathrm{ABK}\left(c \cup \operatorname{PD}\left(\mathcal{A}^{3}\right)\right)} \\
& \cdot \frac{1}{2^{\left|\pi_{0}\left(M^{4}\right)\right|}} \sum_{\substack{a \in C^{1}\left(M^{4}, \mathbb{Z}_{2}\right), b \in \mathcal{C}^{2}\left(M^{4}, \mathbb{Z}_{2}\right)}}(-1) \int_{M^{4}} a\left(\delta b+\mathcal{A}^{3}\right) \\
& \cdot \mathrm{e}^{\frac{2 \pi i}{8} \mathrm{ABK}\left(c \cup \mathrm{PD}^{\prime}(b)\right)} .
\end{aligned}
$$

We write the $\bmod 2$ cohomology class $\mathbb{Z}_{2}$ gauge field as $\mathcal{A} \equiv \mathcal{A}_{\mathbb{Z}_{2}} \equiv\left(\mathcal{A}_{\mathbb{Z}_{4}} \bmod 2\right) \in H^{1}\left(M^{5}, \mathbb{Z}_{2}\right)$. Here come some remarks about this 5D bulk ITQFT-4D boundary TQFT partition function (3.18):

(1) The $\left(\operatorname{PD}\left(\mathcal{A}^{3}\right)\right)$ is a $2 \mathrm{D}$ manifold taking the Poincaré dual (PD) of three-cocycle $\mathcal{A}^{3}$ in the $M^{5}$; but the 2D manifold $\left(\operatorname{PD}\left(\mathcal{A}^{3}\right)\right)$ may touch the the $4 \mathrm{D}$ boundary $\partial M^{5}=M^{4}$. The $1 \mathrm{D}$ boundary $\partial\left(\operatorname{PD}\left(\mathcal{A}^{3}\right)\right)$ can be regarded as the $1 \mathrm{D}$ intersection between the $2 \mathrm{D}$ $\left(\operatorname{PD}\left(\mathcal{A}^{3}\right)\right)$ and the $M^{4}$.

(2) More precisely, for a Spin $\times_{\mathbb{Z}_{2}} \mathbb{Z}_{4}$ manifold $M^{5}$ with a boundary, we have used the Poincare-Lefschetz duality for a manifold with boundaries:

$$
\mathcal{A}^{3} \in H^{3}\left(M^{5}, \mathbb{Z}_{2}\right) \stackrel{\cong}{\rightrightarrows} H_{2}\left(M^{5}, M^{4}, \mathbb{Z}_{2}\right) \ni \operatorname{PD}\left(\mathcal{A}^{3}\right) .
$$

(3) For any pair $\left(S, S^{\prime}\right)$, where $S^{\prime}$ is a subspace of $S$, the short exact sequence of chain complexes

$$
0 \rightarrow C_{*}\left(\mathrm{~S}^{\prime}\right) \rightarrow C_{*}(\mathrm{~S}) \rightarrow C_{*}\left(\mathrm{~S}, \mathrm{~S}^{\prime}\right) \rightarrow 0
$$

with $C_{n}\left(\mathrm{~S}, \mathrm{~S}^{\prime}\right) \equiv C_{n}(\mathrm{~S}) / C_{n}\left(\mathrm{~S}^{\prime}\right)$, induces a long exact sequence of homology groups

$$
\begin{aligned}
\cdots & \rightarrow H_{n}\left(\mathrm{~S}^{\prime}\right) \rightarrow H_{n}(\mathrm{~S}) \rightarrow H_{n}\left(\mathrm{~S}, \mathrm{~S}^{\prime}\right) \\
& \stackrel{\partial}{\rightarrow} H_{n-1}\left(\mathrm{~S}^{\prime}\right) \rightarrow \cdots .
\end{aligned}
$$

Here $H_{n}\left(\mathrm{~S}, \mathrm{~S}^{\prime}\right)$ is the relative homology group, and $\partial$ is the boundary map. (i) Take $\left(\mathrm{S}, \mathrm{S}^{\prime}\right)=\left(\operatorname{PD}\left(\mathcal{A}^{3}\right), \partial \mathrm{PD}\left(\mathcal{A}^{3}\right)\right)$, we denote the boundary map by $\partial$ :

$$
\mathrm{H}_{2}\left(\mathrm{PD}\left(\mathcal{A}^{3}\right), \partial \mathrm{PD}\left(\mathcal{A}^{3}\right)\right) \stackrel{\partial}{\rightarrow} H_{1}\left(\partial \mathrm{PD}\left(\mathcal{A}^{3}\right)\right)
$$

Here $\operatorname{PD}\left(\mathcal{A}^{3}\right)$ is not a closed two manifold, but it has a boundary closed one-manifold $\partial \operatorname{PD}\left(\mathcal{A}^{3}\right)$.

(ii) Take $\left(\mathrm{S}, \mathrm{S}^{\prime}\right)=\left(M^{4}=\partial M^{5}, \partial \mathrm{PD}\left(\mathcal{A}^{3}\right)\right)$, we denote another boundary map by $\partial_{1}$ :

$$
\mathrm{H}_{2}\left(M^{4}, \partial \mathrm{PD}\left(\mathcal{A}^{3}\right)\right) \stackrel{\partial_{1}}{\rightarrow} \mathrm{H}_{1}\left(\partial \mathrm{PD}\left(\mathcal{A}^{3}\right)\right)
$$

Both $M^{4}=\partial M^{5}$ and $\partial \operatorname{PD}\left(\mathcal{A}^{3}\right)$ are closed manifolds, of $4 \mathrm{D}$ and $1 \mathrm{D}$, respectively.

(4) Now, the $c$ is defined as a 2D surface living on the boundary $M^{4}$. The $\partial_{1} c$ uses the boundary map (3.23)'s $\partial_{1}$ of $c$ on the $M^{4}$. We can compensate the two-surface $\operatorname{PD}\left(\mathcal{A}^{3}\right)$ potentially with a one boundary, by gluing it with $c$ to make a closed two surface. To do so, we require both $\operatorname{PD}\left(\mathcal{A}^{3}\right)$ and $c$ share the same 1D boundary.

(5) The $c \in \partial_{1}{ }^{-1}\left(\partial\left[\operatorname{PD}\left(\mathcal{A}^{3}\right)\right]\right)$ also means

$$
\partial_{1} c=\partial\left[\mathrm{PD}\left(\mathcal{A}^{3}\right)\right]=\left[\partial \mathrm{PD}\left(\mathcal{A}^{3}\right)\right]
$$

(i) The $\left[\operatorname{PD}\left(\mathcal{A}^{3}\right)\right]$ means the fundamental class and the relative homology class of the $2 \mathrm{D}$ manifold $\operatorname{PD}\left(\mathcal{A}^{3}\right)$.

(ii) The $\partial\left[\mathrm{PD}\left(\mathcal{A}^{3}\right)\right]=\left[\partial \mathrm{PD}\left(\mathcal{A}^{3}\right)\right]$ means the boundary of the fundamental class (via the boundary $\partial$ map in (3.22)) is equivalent to the fundamental class of the boundary $\partial$ of $\operatorname{PD}\left(\mathcal{A}^{3}\right)$. Beware that the two $\partial$ operations in $\partial\left[\operatorname{PD}\left(\mathcal{A}^{3}\right)\right]=\left[\partial \mathrm{PD}\left(\mathcal{A}^{3}\right)\right]$ have different meanings.

(iii) Equation (3.24) exactly matches the requirement that the bulk two-surface $\operatorname{PD}\left(\mathcal{A}^{3}\right)$ (living in $M^{5}$ ) and the boundary two-surface $c$ (living on $M^{4}$ ) share the same 1D boundary.

(6) Note that when $M^{5}$ is a closed manifold with no boundary $M^{4}=\partial M^{5}=\varnothing$ thus $c=\varnothing$, then the term

$$
\sum_{c \in \partial_{1}^{-1}\left(\partial\left[\operatorname{PD}\left(\mathcal{A}^{3}\right)\right]\right)} \mathrm{e}^{\frac{2 \pi \mathrm{i}}{8} \mathrm{ABK}\left(c \cup \operatorname{PD}\left(\mathcal{A}^{3}\right)\right)} \text { is equivalently reduced to } \mathrm{e}^{\frac{2 \pi \mathrm{i}}{8} \mathrm{ABK}\left(\operatorname{PD}\left(\mathcal{A}^{3}\right)\right)}=\mathbf{Z}_{5 \mathrm{D}-\mathrm{TTQFT}}^{\left(\nu_{\text {even }}=2\right)}
$$

\footnotetext{
${ }^{21}$ We use the $\smile$ notation for the cup product between cohomology classes, or between a cohomology class and a fermionic topological invariant (paired via a Poincaré dual PD). We use the $U$ notation for the surgery gluing the boundaries of two manifolds within relative homology classes. So the $M_{1} \cup M_{2}$ means gluing the boundary $\partial M_{1}=\overline{\partial M_{2}}$ such that the common orientation of $\overline{\partial M_{2}}$ is the reverse of $\partial M_{2}$.
} 
This is a satisfactory consistent check, consistent with the 5D bulk-only ITQFT at $\nu_{\text {even }}=2$ in (3.16).

(7) The $a \in C^{1}\left(M^{4}, \mathbb{Z}_{2}\right)$ means that $a$ is a onecochain, and the $b \in C^{2}\left(M^{4}, \mathbb{Z}_{2}\right)$ means that $b$ is a two-cochain. The factor $(-1)^{\int_{M^{4}} a\left(\delta b+\mathcal{A}^{3}\right)}=$ $\exp \left(\mathrm{i} \pi \int_{M^{4}} a\left(\delta b+\mathcal{A}^{3}\right)\right)$ gives the weight of the $4 \mathrm{D}$ $\mathbb{Z}_{2}$ gauge theory. The $a \delta b$ term is the level-two BF theory written in the mod 2 class. ${ }^{22}$ The path integral sums over these distinct cochain classes.

The variation of $a$ gives the equation of motion $\left(\delta b+\mathcal{A}^{3}\right)=0 \bmod 2$. In the path integral, we can integrate out $a$ to give the same constraint $\left(\delta b+\mathcal{A}^{3}\right)=0 \bmod 2$. This is precisely the trivialization of the second cohomology class,

$$
\mathcal{A}^{3}=\left(\mathcal{A}_{\mathbb{Z}_{2}}\right)^{3}=\delta b \quad \bmod 2,
$$

so thethree-cocycle becomes a three-coboundary that splits to a two-cochain $b$. This exactly matches the condition imposed by the symmetry extension (3.17): $1 \rightarrow\left[\mathbb{Z}_{2}\right] \rightarrow \operatorname{Spin} \times \mathbb{Z}_{4, X} \rightarrow$ Spin $\times_{\mathbb{Z}_{2}^{F}} \mathbb{Z}_{4, X} \rightarrow 1$,

where the $\mathcal{A}^{3}$ term in Spin $\times_{\mathbb{Z}_{2}^{F}} \mathbb{Z}_{4, X}$ becomes trivialized as a coboundary $\mathcal{A}^{3}=\delta b$ (so $\mathcal{A}^{3}=0$ in terms of a cohomology or cocycle class) in Spin $\times \mathbb{Z}_{4, X}$.

(8) The $\frac{1}{2 \pi_{0}\left(M^{4}\right) \mid}$ factor mod out the gauge redundancy for the boundary $4 \mathrm{D} \mathbb{Z}_{2}$ gauge theory. The $\pi_{0}\left(M^{4}\right)$ is the zeroth homotopy group of $M^{4}$, namely the set of all path components of $M^{4}$. Thus, we only sum over the gauge equivalent classes in the path integral.

(9) The $\operatorname{ABK}\left(c \cup \operatorname{PD}\left(\mathcal{A}^{3}\right)\right)$ is defined on a $2 \mathrm{D}$ manifold with $\mathrm{Pin}^{-}$structure. Recall the $M^{5}$ has the Spin $\times_{\mathbb{Z}_{2}} \mathbb{Z}_{4}$ structure. If $M^{5}$ is closed, then there is a natural Smith map to induce the $2 \mathrm{D} \mathrm{Pin}^{-}$

\footnotetext{
${ }^{22}$ The continuum QFT version of this $\mathbb{Z}_{2}$ gauge theory is $\left.\exp \left(\mathrm{i} \int_{M^{4}} \frac{2}{2 \pi} a \mathrm{~d} b+\frac{1}{\pi^{3}} a \mathcal{A}^{3}\right)\right)$, where the $b$ integration over a closed two cycle, $\$ b$, can be $n \pi$ with some integer $n \in \mathbb{Z}$. The $a$ and $\mathcal{A}$ integration over a closed one cycle, $\oint a$ and $\oint \mathcal{A}$, can be $n \pi$ with some integer $n \in \mathbb{Z}$. Another alternative possibility of 4D TQFT of (3.18) can be $\frac{1}{2^{\left|\pi_{0}\left(M^{4}\right)\right|}} \sum_{a \in C^{1}\left(M^{4}, \mathbb{Z}_{2}\right), b \in C^{2}\left(M^{4}, \mathbb{Z}_{2}\right)}(-1) \int_{M^{4}} b\left(\delta a+\mathcal{A}^{2}\right)$.

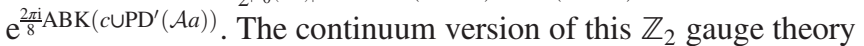
has a different expression as $\left.\exp \left(i \int_{M^{4}} \frac{2}{2 \pi} b \mathrm{~d} a+\frac{1}{\pi^{2}} b \mathcal{A}^{2}\right)\right)$. Either $4 \mathrm{D} \mathbb{Z}_{2}$ gauge theory sits at the normal subgroup $\left[\mathbb{Z}_{2}\right]$ of the group extension (3.17). Although the $\left[\mathbb{Z}_{2}\right]$ is abelian, this 4D TQFT actually exhibits non-abelian topological order due to the fermionic nature of Spin $\times_{\mathbb{Z}_{2}^{F}} \mathbb{Z}_{4, X}$ and the fermionic invariant ABK. The non-abelian nature of this $4 \mathrm{~d}$ TQFT is similar to the non-abelian nature of $3 d \mathbb{Z}_{2}$ gauge theory obtained from gauging the $\mathbb{Z}_{2}$-onsite symmetry of the odd class of $2+1 \mathrm{~d}$ fermionic topological superconductor from the $\Omega_{3}^{\operatorname{Spin} \times \mathbb{Z}_{2}}=\mathbb{Z}_{8}$ classification (e.g., Section 8 of [58], and [47]). The braiding and fusions statistics of vortices of these TQFTs are non-abelian. Moreover, we may require additional symmetry extension beyond (3.17) to construct 4D TQFTs.
}

structure on the closed surface via $\operatorname{PD}\left(\mathcal{A}^{3}\right)$. However, the $M^{5}$ has a boundary $M^{4}$, so $\operatorname{PD}\left(\mathcal{A}^{3}\right)$ may not be closed-the previously constructed closed two surface $\left(c \cup \operatorname{PD}\left(\mathcal{A}^{3}\right)\right)$ is meant to induce a $2 \mathrm{D}$ Pin $^{-}$structure. ${ }^{23}$ Then we compute the ABK on this closed two surface $\left(c \cup \operatorname{PD}\left(\mathcal{A}^{3}\right)\right)$.

(10) Let us explain the other term $\operatorname{ABK}\left(c \cup \operatorname{PD}^{\prime}(\mathcal{A} a)\right)$ of the 4D boundary TQFT in (3.18). The PD' is the Poincaré dual on $M^{4}=\partial M^{5}$. Since the fundamental classes of $M^{5}$ and $M^{4}=\partial M^{5}$ are related by

$$
\begin{aligned}
& {\left[M^{5}\right] \in H_{5}\left(M^{5}, M^{4}, \mathbb{Z}_{2}\right)} \\
& \quad \stackrel{\partial}{\rightarrow} H_{4}\left(M^{4}, \mathbb{Z}_{2}\right) \ni\left[M^{4}\right] \equiv\left[\partial M^{5}\right] .
\end{aligned}
$$

Here we have the following relations: ${ }^{24}$

$$
\begin{aligned}
& \mathrm{PD}=\left[M^{5}\right] \cap, \\
& \begin{aligned}
\mathrm{PD}^{\prime}=\left[M^{4}\right] \cap=\left[\partial M^{5}\right] \cap=\partial\left[M^{5}\right] \cap, \\
\partial^{\prime} \mathrm{PD}^{\prime}(b)=\operatorname{PD}^{\prime}(\delta b)=\operatorname{PD}^{\prime}\left(\mathcal{A}^{3}\right) \\
=\partial\left[\operatorname{PD}\left(\mathcal{A}^{3}\right)\right]=\partial_{1} c .
\end{aligned}
\end{aligned}
$$

(i) The cap product $\cap$ here is to define $\mathrm{PD}$ homology class, such that $\operatorname{PD}(\mathcal{A})=\left[M^{5}\right] \cap \mathcal{A}$.

(ii) Here we use $\left[M^{4}\right]=\left[\partial M^{5}\right]=\partial\left[M^{5}\right]$ : the fundamental class of boundary of $M^{5}$ gives the boundary of fundamental class.

(iii) Equation (3.27)'s first equality $\partial^{\prime} \mathrm{PD}^{\prime}(b)=$ $\mathrm{PD}^{\prime}(\delta b)$ uses the coboundary operator $\delta$ on the cohomology class $b$.

(iv) Equation (3.27)'s second equality $\operatorname{PD}^{\prime}(\delta b)=$ $\operatorname{PD}^{\prime}\left(\mathcal{A}^{3}\right)$ uses the condition $\delta \mathcal{A}=0$ and the trivialization condition (3.25): $\delta b=\mathcal{A}^{3}$.

(v) Equation (3.27)'s third equality $\operatorname{PD}^{\prime}\left(\mathcal{A}^{3}\right)=$ $\partial\left[\operatorname{PD}\left(\mathcal{A}^{3}\right)\right]$, we use "the naturality of the cap product." There are natural pushforward and pullback maps on homology and cohomology,

\footnotetext{
${ }^{23} \mathrm{We}$ do not yet know whether it is always possible to induce a unique 2D $\mathrm{Pin}^{-}$structure on $\left(c \cup \operatorname{PD}\left(\mathcal{A}^{3}\right)\right)$ for any possible pair of data $\left(M^{5}, M^{4}=\partial M^{5}\right)$ given any $M^{5}$ with $\operatorname{Spin} \times_{\mathbb{Z}_{2}} \mathbb{Z}_{4}$ structure. However, we claim that it is possible to find some suitable $M^{4}$ so that the $2 \mathrm{D}\left(c \cup \operatorname{PD}\left(\mathcal{A}^{3}\right)\right)$ has $\mathrm{Pin}^{-}$induced, thus in this sense the $\operatorname{ABK}\left(c \cup \operatorname{PD}\left(\mathcal{A}^{3}\right)\right)$ is defined. For physics purposes, it is enough that we can firstly focus on studying the theory on these types of $\left(M^{5}, M^{4}=\partial M^{5}\right)$.

${ }^{24}$ Let us clarify the notations: $\partial, \partial^{\prime}$, and $\partial_{1}$. The boundary notation $\partial$ may mean as (1) taking the boundary, or (2) in the boundary map of relative homology class in (3.22). It should be also clear to the readers that the $\partial$ is associated with the operations on objects living in the bulk $M^{5}$ or ending on the boundary $M^{4}$ and while $\partial^{\prime}$ is associated with the operations on objects living on the boundary $M^{4}$ alone. The $\partial_{1}$ is defined as another boundary map in (3.23).
} 
related by the projection formula, also known as "the naturality of the cap product."

(vi) Equation (3.27)'s last equality $\partial\left[\operatorname{PD}\left(\mathcal{A}^{3}\right)\right]=$ $\partial_{1} c$ is based on (3.24). Importantly, as a satisfactory consistency check, this also shows that the two-surface $c$ obeys the following:

the $c$ in $\operatorname{ABK}\left(c \cup \operatorname{PD}\left(\mathcal{A}^{3}\right)\right)$ is the same $c$ in

$\operatorname{ABK}\left(c \cup \operatorname{PD}^{\prime}(b)\right)$.

(vii) As before, the union $\left(c \cup \mathrm{PD}^{\prime}(b)\right)$ is a closed two-surface and we induce a $\mathrm{Pin}^{-}$structure on this two surface. So we can compute the ABK on this closed two surface $\left(c \cup \mathrm{PD}^{\prime}(b)\right)$ on the $M^{4}{ }^{25}$

In summary, we have constructed the $4 \mathrm{D} \mathbb{Z}_{2}$-gauge theory in (3.18) preserving the $\left(\operatorname{Spin} \times_{\mathbb{Z}_{2}^{F}} \mathbb{Z}_{4, X}\right)$ structure, namely it is a $\left(\operatorname{Spin} \times_{\mathbb{Z}_{2}^{F}} \mathbb{Z}_{4, X}\right)$-symmetric TQFT but with $\nu_{\text {even }}=2 \in \mathbb{Z}_{16}$ anomaly. This can be used to compensate the anomaly $\nu=-N_{\text {generation }} \bmod 16$ with $N_{\text {generation }}=2$, two generations of missing right-handed neutrinos. We could not however directly construct the symmetric gapped TQFT for $\nu$ is odd (thus symmetric TQFTs not possible for $N_{\text {generation }}=1$ or 3 ), due to the obstruction found in $[46,83]$.

\section{General principle}

The discussion in Sec. III C 1 says that only for the even integer $\nu_{4 \mathrm{D} \text {,even }} \in \mathbb{Z}_{16}$ does the $\left(\operatorname{Spin} \times_{\mathbb{Z}_{2}^{F}} \mathbb{Z}_{4, X}\right)$-symmetrypreserving TQFT exist. This prompts us to improve the SM version (3.12) into

$$
\begin{aligned}
& \mathbf{Z}_{\mathrm{UU}}\left[\mathcal{A}_{\mathbb{Z}_{4}}\right] \equiv \mathbf{Z}_{\substack{\text { SD-ITOFT/ } \\
4 \mathrm{SM}+\mathrm{TQFT}}}\left[\mathcal{A}_{\mathbb{Z}_{4}}\right] \\
& \equiv \mathbf{Z}_{5 \mathrm{D}-\mathrm{ITQFT}}^{\left(-\nu_{5 \mathrm{D}}\right)}\left[\mathcal{A}_{\mathbb{Z}_{4}}\right] \cdot \mathbf{Z}_{4 \mathrm{D} \text {-TQFT }}^{\left(\nu_{4 \mathrm{D} \text {,even }}\right)}\left[\mathcal{A}_{\mathbb{Z}_{4}}\right] \\
& \cdot \mathbf{Z}_{\mathrm{SM}}^{\left(n_{\nu_{e, R}}, n_{\nu_{\mu, R}}, n_{\nu_{\tau, R}}\right)}\left[\mathcal{A}_{\mathbb{Z}_{4}}\right] .
\end{aligned}
$$

The GUT version (3.13) should be adjusted into

$$
\begin{aligned}
& \mathbf{Z}_{\mathrm{UU}}\left[\mathcal{A}_{\mathbb{Z}_{4}}\right] \equiv \mathbf{Z}_{\substack{\text { SD-ITOFT/ } \\
\text { SD-GTT }+\mathrm{TPFT}}}\left[\mathcal{A}_{\mathbb{Z}_{4}}\right] \\
& \equiv \mathbf{Z}_{5 \mathrm{D} \text {-ITQFT }}^{\left(-\nu_{5 \mathrm{D}}\right)}\left[\mathcal{A}_{\mathbb{Z}_{4}}\right] \cdot \mathbf{Z}_{4 \mathrm{D} \text {-TQFT }}^{\left(\nu_{4 \mathrm{D} \text {,even }}\right)}\left[\mathcal{A}_{\mathbb{Z}_{4}}\right] \\
& \cdot \mathbf{Z}_{\mathrm{GUT}}^{\left(n_{\nu_{e, R}}, n_{\nu_{\mu, R}}, n_{\nu_{\tau, R}}\right)}\left[\mathcal{A}_{\mathbb{Z}_{4}}\right] \text {. }
\end{aligned}
$$

Also the anomaly constraint (2.11) becomes

\footnotetext{
${ }^{25}$ Similar to footnote 24 , we can find some suitable $M^{4}$ so that the $2 \mathrm{D} \mathrm{Pin}^{-}$is induced, thus in this sense the $\operatorname{ABK}\left(c \cup \mathrm{PD}^{\prime}(b)\right)$ is defined.
}

$$
\begin{aligned}
& \left(-\left(N_{\mathrm{gen}}=3\right)+n_{\nu_{e, R}}+n_{\nu_{\mu, R}}+n_{\nu_{\tau, R}}+\nu_{4 \mathrm{D}, \text { even }}-\nu_{5 \mathrm{D}}\right) \\
& \quad=0 \bmod 16 .
\end{aligned}
$$

This implies that the existence of symmetry-preserving 4D TQFT sector requires the following ${ }^{26}$ :

$$
\left(n_{\nu_{e, R}}+n_{\nu_{\mu, R}}+n_{\nu_{\tau, R}}-\nu_{5 \mathrm{D}}\right) \text { must be an odd integer. }
$$

Now we have derived an ultra unification path integral in (3.30) and (3.31), including 4D SM (3.1), 4D GUT (3.8), 5D ITQFT (3.15), and 4D TQFT (3.18), comprising many scenarios and their linear combinations enlisted in Sec. II C: (1a), (1b), (1c), (2a), and (2b). Then we can dynamically gauge the appropriate bulk-boundary global symmetries, promoting the theory to a bulk gauge theory in scenarios (2c) and (2d), or break some of the (global or gauge) symmetries to (2e).

In summary, we propose a general principle behind the ultra unification:

(1) We start with a QFT in general as an EFT given some full spacetime-internal symmetry $G$, say in a $D$ dimensional spacetime.

(2) We check the anomaly and cobordism constraint given by $G$ via computing $\Omega_{G}^{\mathrm{D}+1} \equiv \mathrm{TP}_{\mathrm{D}+1}(G)$.

(3) We check the anomaly index of the $D$ dimensional QFT/EFT constrained by cobordism $\Omega_{G}^{\mathrm{D}+1} \equiv \mathrm{TP}_{\mathrm{D}+1}(G)$.

(4) If all anomalies are cancelled, then we do not require any new hidden sector to define $D$-dimensional QFT/EFT.

(5) If some anomalies are not cancelled, then either

(i) We need to break some symmetry out of $G$, or

\footnotetext{
${ }^{26}$ The nonperturbative global anomaly cancellation constraint $\left(-\left(N_{\text {gen }}=3\right)+n_{\nu_{e, R}}+n_{\nu_{\mu, R}}+n_{\nu_{\tau, R}}+\nu_{4 \mathrm{D}, \text { even }}-\nu_{5 \mathrm{D}}\right)=0 \bmod 16$ provides the capacity for many kinds of the ultra unification model building. For example, the $4 \mathrm{D} \mathbb{Z}_{4, X}$-symmetry preserving TQFT sector can take the index $\nu_{4 \mathrm{D} \text {,even }}=0,2,4, \ldots$ for any even integer. There are many (perhaps, infinite) types of TQFTs for each index $\nu_{4 \mathrm{D}, \text { even }}$. But to be more economic, we can ask for the minimum degrees of freedom required by a TQFT for any given index $\nu_{4 \mathrm{D} \text {,even. }}$ Also for HEP phenomenological purposes, by taking account of the experimentally observed neutrino mass eigenstates splitting, one may propose to have at least two generations of right-handed neutrinos, which means that a possible phenomenological input $n_{\nu_{e, R}}+n_{\nu_{\mu, R}}+n_{\nu_{\tau, R}} \geq 2$. In summary, a viable ultra unification candidate can be, for example, $n_{\nu_{e, R}}+n_{\nu_{\mu, R}}+n_{\nu_{\tau, R}}=2, \nu_{4 \mathrm{D} \text {,even }}=2$, and $\nu_{5 \mathrm{D}}=1$, which saturate the anomaly cancellation and some phenomenological constraints. So far, we mainly use the cobordism theory to study the invertible anomalies and invertible topological field theories, and we also use the cohomology data to construct noninvertible topological quantum field theories. However, once the discrete symmetries (such as $\mathbb{Z}_{4, X}$ ) are dynamically gauged, it is more natural to use the mathematical category or higher category theories to characterize the topological phase sectors.
} 
(ii) We extend the symmetry $G$ to an appropriate $\tilde{G}$ to trivialize nonperturbative global anomalies in $\Omega_{\tilde{G}}^{\mathrm{D}+1} \equiv \mathrm{TP}_{\mathrm{D}+1}(\tilde{G})$, or

(iii) We propose new hidden sectors appending to Dd QFT/EFT, with a schematic path integral (say if we add Dd-TQFT or CFT and $(D+1)$-dimensional ITQFT onto the original theory):

$$
\mathbf{Z}_{\substack{(D+1) \text { D-ITPFT/ } \\ \text { DdFT/EFT+TQFT r CFT }}}[\mathcal{A}] \equiv \mathbf{Z}_{(D+1) \text { D-ITQFT }}[\mathcal{A}] \cdot \mathbf{Z}_{\text {Dd-TQFT or CFT }}[\mathcal{A}] \cdot \mathbf{Z}_{\text {Dd QFT/EFT }}[\mathcal{A}] \cdot
$$

\section{Detect topological phase sectors and the essence}

\section{Detect Topological Forces}

By looking at Fig. 1, in the Higgs vacuum where our SM EFT resides in, we have only detected the strong, electromagnetic, and weak in the subatomic physics. The GUT forces are weaker than the weak force, and the topological force is further weaker than the GUT and weak forces. So how could we experimentally detect topological forces?

Notice that the gravity is further weaker than all other forces. (So how could we experimentally detect gravity?) But the gravity has accumulative effects that only have the gravitational attractions. Without doubt, the gravity has been detected by everyone and by all astrophysics and cosmology observations. The gravity had been detected first in the human history among all the forces!

Similarly, although topological force is also weak (but stronger than the gravity), topological force is infinite range or long range that does not decay in the long distance, and it mediates between the linked worldline/world sheet/world volume trajectories of the charged (pointlike or extended) objects via fractional or categorical anyonic statistical interactions. So, in principle, we may have already experienced topological force in our daily life in a previously scientifically unnoticed way. ${ }^{27}$

\section{Neutrino oscillations and dark matter}

In fact, Ref. [9] had proposed that the topological force may cause (thus be detected by) the phenomena of neutrino oscillations. We can consider the Majorana zero modes of the vortices in the $4 \mathrm{D}$ TQFT defects. The left-handed neutrinos (confirmed by experiments) are

\footnotetext{
${ }^{27}$ For example, if the $\mathbb{Z}_{4, X}$ is dynamically gauged, there is a dynamical discrete gauge Wilson line connecting all SM fermions living in 4d SM or GUT (e.g., quarks and electrons in our body). Namely, the SM fermions can live at the open ends of the $\mathbb{Z}_{4, X}$ gauged Wilson line. Thus there could be long-distance topological interactions and communications between the $\mathbb{Z}_{4, X}$-gauge charged objects. Moreover, the $\mathbb{Z}_{4, X}$ gauged Wilson line as a $\mathcal{A}_{\mathbb{Z}_{4}}$ gauge field on the $4 \mathrm{~d}$ theory can be leaked into the $5 \mathrm{~d}$ bulk theory as a $\mathcal{A}_{\mathbb{Z}_{2}}$ gauge field. In the $5 \mathrm{~d}$ bulk, a nontrivial link configuration can be charged under the other end of $\mathcal{A}_{\mathbb{Z}_{2}}$ [9]. In any case, every phenomenon and every law of Nature should be explained by mathematics and physics principles.
}

nearly gapless/ massless. When the left-handed neutrinos traveling through the 4D TQFT defects, we may observe nearly gapless neutrino flavor oscillations interfering with the Majorana zero modes trapped by the vortices in the $4 \mathrm{D}$ TQFT defects.

On the other hand, the gapped heavy excitations (point or extended objects) of topological phase sector may be a significant contribution to dark matter [86].

\section{The essence of ultra unification}

Finally, we come to the essence of ultra unification. What is unified after all? We have united the strong, electromagnetic, weak, GUT forces, and topological forces into the same theory in the ultra unification QFT/TQFT path integral (that this theory can also be coupled to the curved spacetime geometry and gravity and at least well defined in a background nondynamical way).

However, the grand unification $[6,7]$ united the three gauge interactions of the SM into a single electronuclear force under a simple Lie group gauge theory. Do we have any equivalent statement to also unite strong, electromagnetic, weak, GUT forces, and topological forces into a single force at a high enough energy? We believe that the definite answer relies on studying the details of analogous topological quantum phase transitions $[10,86]$ and the parent effective field theory that describes the phase transition and neighborhood phases, such as those explored in $4 d$ [65,87-90]. The underlying mathematical structure suggests a $4 \mathrm{~d}$ version of particle-vortex duality, S-duality, T-duality, or mirror symmetry.

\section{E. Summary of ultra unification and quantum matter}

Let us summarize what we have done in this work in a Quantum Matter perspective:

(i) We have started from the Nature given Standard Model (SM) quarks and leptons, and their quantum numbers, in three generations.

(ii) We have included gauge forces and (various) Higgs for SM and Grand Unification (GUT).

(iii) After the essential check of the anomaly matching and cobordism constraints, the detection of the $\mathbb{Z}_{16}$ global anomaly for $15 n$ Weyl fermion SM and GUT implies that we can choose (as one of many options) to realize our $4 \mathrm{~d}$ world living on an extra dimensional 5D invertible TQFT (a 5D 
topological superconductor, mathematically a 5D cobordism invariant). It may be understood as a one-brane 4D world with an extra large fifth dimension.

(iv) Ultra Unification incorporates the SM, GUT, and Topological forces into the same theory (that this theory can also be coupled to curved spacetime geometry and gravity in a background non-dynamical way). It may also be understood as a multi-branes or two-brane 4D world with an extra fifth dimension. The issues of mirror fermion doubling [91] on the mirror world, depending on the precise anomaly index on the mirror sector, may be fully trivially gapped (if anomaly-free), may contain a mirror chiral gauge theory or unparticle conformal field theory, or may be topological order gapped with a low energy TQFT. These issues are tackled in many recent works [13,92-102].

(v) In Quantum Matter terminology, we show that SM and GUT belong to a framework of a continuous gauge field theory, Anderson-Higgs (global or gauge) symmetry-breaking mass, and Ginzburg-Landau paradigm. In contrast, the new sectors that we introduce are beyond Ginzburg-Landau paradigm. The new sectors include a fermionic discrete gauge theory, symmetry-extension topological mass, and modern issues on symmetry, topology, nonperturbative interactions, and short/long-range entanglements.

\section{ACKNOWLEDGMENTS}

J.W. thanks the participants of Quantum Matter in Mathematics and Physics program at Harvard University CMSA for the enlightening atmosphere. Part of this work had been presented at Higher Structures and Field Theory at Erwin Schrödinger Institute in Wien [103], and at Harvard University Particle Physics Lunch seminar and String Lunch seminar. J. W. thanks the valuable feedbacks from the seminar participants. This work is supported by NSF Grant No. DMS-1607871 "Analysis, Geometry and Mathematical Physics" and the Center for Mathematical Sciences and Applications at Harvard University.
[1] J. C. Maxwell, A dynamical theory of the electromagnetic field, Phil. Trans. R. Soc. London 155, 459 (1865).

[2] S. L. Glashow, Partial symmetries of weak interactions, Nucl. Phys. 22, 579 (1961).

[3] A. Salam and J.C. Ward, Electromagnetic and weak interactions, Phys. Lett. 13, 168 (1964).

[4] A. Salam, Weak and electromagnetic interactions, Conf. Proc. C680519, 367 (1968).

[5] S. Weinberg, A Model of Leptons, Phys. Rev. Lett. 19, 1264 (1967).

[6] H. Georgi and S. L. Glashow, Unity of All Elementary Particle Forces, Phys. Rev. Lett. 32, 438 (1974).

[7] H. Fritzsch and P. Minkowski, Unified interactions of leptons and hadrons, Ann. Phys. (N.Y.) 93, 193 (1975).

[8] Z. Wan and J. Wang, Beyond standard models and grand unifications: Anomalies, topological terms, and dynamical constraints via cobordisms, J. High Energy Phys. 07 (2020) 062.

[9] J. Wang, Anomaly and cobordism constraints beyond standard model: Topological force, arXiv:2006.16996.

[10] J. Wang, Anomaly and cobordism constraints beyond grand unification: Energy hierarchy, arXiv:2008.06499.

[11] D. S. Freed, Pions and generalized cohomology, J. Diff. Geom. 80, 45 (2008).

[12] I. Garcia-Etxebarria and M. Montero, Dai-Freed anomalies in particle physics, J. High Energy Phys. 08 (2019) 003.

[13] J. Wang and X.-G. Wen, A non-perturbative definition of the standard models, Phys. Rev. Research 2, 023356 (2020).

[14] J. Davighi, B. Gripaios, and N. Lohitsiri, Global anomalies in the standard model(s) and beyond, arXiv:1910.11277.
[15] J.F. Adams, On the structure and applications of the Steenrod algebra, Commentarii mathematici Helvetici 32, 180 (1958).

[16] R. Thom, Quelques propriétés globales des variétés différentiables, Commentarii mathematici Helvetici 28, 17 (1954).

[17] S. Galatius, I. Madsen, U. Tillmann, and M. Weiss, The homotopy type of the cobordism category, Acta Math. 202, 195 (2009).

[18] D. S. Freed and M. J. Hopkins, Reflection positivity and invertible topological phases, arXiv:1604.06527.

[19] G. 't Hooft, Naturalness, chiral symmetry, and spontaneous chiral symmetry breaking, NATO Sci. Ser. B 59, 135 (1980).

[20] S. L. Adler, Axial vector vertex in spinor electrodynamics, Phys. Rev. 177, 2426 (1969).

[21] J. S. Bell and R. Jackiw, A PCAC puzzle: $\pi^{0} \rightarrow \gamma \gamma$ in the $\sigma$ model, Nuovo Cimento A 60, 47 (1969).

[22] L. Alvarez-Gaume and E. Witten, Gravitational anomalies, Nucl. Phys. B234, 269 (1984).

[23] E. Witten, An SU(2) anomaly, Phys. Lett. 117B, 324 (1982).

[24] J. Wang, X.-G. Wen, and E. Witten, A new SU(2) anomaly, J. Math. Phys. (N.Y.) 60, 052301 (2019).

[25] E. Witten, Global gravitational anomalies, Commun. Math. Phys. 100, 197 (1985).

[26] Z. Wan and J. Wang, Higher anomalies, higher symmetries, and cobordisms I: Classification of highersymmetry-protected topological states and their boundary fermionic/bosonic anomalies via a generalized cobordism theory, Ann. Math. Sci. Appl. 4, 107 (2019). 
[27] E. Witten and K. Yonekura, Anomaly inflow and the $\eta$-invariant, in The Shoucheng Zhang Memorial Workshop Stanford, CA, USA, 2019 (2019), https://arxiv.org/abs/ 1909.08775.

[28] S.-S. Chern and J. Simons, Characteristic forms and geometric invariants, Ann. Math. 99, 48 (1974).

[29] A. S. Schwarz, The partition function of degenerate quadratic functional and Ray-Singer invariants, Lett. Math. Phys. 2, 247 (1978).

[30] E. Witten, Quantum field theory and the Jones polynomial, Commun. Math. Phys. 121, 351 (1989).

[31] X.-G. Wen, Zoo of quantum-topological phases of matter, Rev. Mod. Phys. 89, 041004 (2017).

[32] T. Senthil, Symmetry-protected topological phases of quantum matter, Annu. Rev. Condens. Matter Phys. 6, 299 (2015).

[33] M.Z. Hasan and C.L. Kane, Colloquium: Topological insulators, Rev. Mod. Phys. 82, 3045 (2010).

[34] X.-L. Qi and S.-C. Zhang, Topological insulators and superconductors, Rev. Mod. Phys. 83, 1057 (2011).

[35] O. Aharony, N. Seiberg, and Y. Tachikawa, Reading between the lines of four-dimensional gauge theories, J. High Energy Phys. 08 (2013) 115.

[36] D. Tong, Line operators in the standard model, J. High Energy Phys. 07 (2017) 104.

[37] J.P. Ang, K. Roumpedakis, and S. Seifnashri, Line operators of gauge theories on non-spin manifolds, J. High Energy Phys. 04 (2020) 087.

[38] Z. Wan, J. Wang, and Y. Zheng, Higher anomalies, higher symmetries, and cobordisms II: Lorentz symmetry extension and enriched bosonic/fermionic quantum gauge theory, Ann. Math. Sci. Appl. 5, 171 (2020).

[39] F. Wilczek and A. Zee, Operator Analysis of Nucleon Decay, Phys. Rev. Lett. 43, 1571 (1979).

[40] Z. Wan, J. Wang et al., Higher Anomalies, higher symmetries, and cobordisms IV: Standard models, grand unifications and beyond (to be published).

[41] C. Arf, Untersuchungen über quadratische Formen in Körpern der Charakteristik 2, I, J. Reine Angew. Math. 183, 148 (1941).

[42] A. Y. Kitaev, Unpaired Majorana fermions in quantum wires, Phys. Usp. 44, 131 (2001).

[43] J. Davighi and N. Lohitsiri, Anomaly interplay in $U(2)$ gauge theories, J. High Energy Phys. 05 (2020) 098.

[44] M. F. Atiyah, V. K. Patodi, and I. M. Singer, Spectral asymmetry and Riemannian geometry 1, Math. Proc. Cambridge Philos. Soc. 77, 43 (1975).

[45] Y. Tachikawa and K. Yonekura, Why are fractional charges of orientifolds compatible with Dirac quantization?, SciPost Phys. 7, 058 (2019).

[46] C.-T. Hsieh, Discrete gauge anomalies revisited, arXiv: 1808.02881 .

[47] M. Guo, K. Ohmori, P. Putrov, Z. Wan, and J. Wang, Fermionic finite-group gauge theories and interacting symmetric/crystalline orders via cobordisms, Commun. Math. Phys. 376, 1073 (2020).

[48] A. Kapustin, R. Thorngren, A. Turzillo, and Z. Wang, Fermionic symmetry protected topological phases and cobordisms, J. High Energy Phys. 12 (2015) 052.
[49] I. Hason, Z. Komargodski, and R. Thorngren, Anomaly matching in the symmetry broken phase: Domain walls, $C P T$, and the Smith isomorphism, SciPost Phys. 8, 062 (2020).

[50] A. Vishwanath and T. Senthil, Physics of Three Dimensional Bosonic Topological Insulators: Surface Deconfined Criticality and Quantized Magnetoelectric Effect, Phys. Rev. X 3, 011016 (2013).

[51] J. Wang, X.-G. Wen, and E. Witten, Symmetric Gapped Interfaces of SPT and SET States: Systematic Constructions, Phys. Rev. X 8, 031048 (2018).

[52] H. Georgi, Unparticle Physics, Phys. Rev. Lett. 98, 221601 (2007).

[53] Fractional Statistics and Anyon Superconductivity, edited by F. Wilczek (World Scientific, Singapore, 1990).

[54] C. Wang and M. Levin, Braiding Statistics of Loop Excitations in Three Dimensions, Phys. Rev. Lett. 113, 080403 (2014).

[55] S. Jiang, A. Mesaros, and Y. Ran, Generalized Modular Transformations in $(3+1) \mathrm{D}$ Topologically Ordered Phases and Triple Linking Invariant of Loop Braiding, Phys. Rev. X 4, 031048 (2014).

[56] J. C. Wang and X.-G. Wen, Non-Abelian string and particle braiding in topological order: Modular SL $(3, \mathrm{Z})$ representation and $(3+1)$-dimensional twisted gauge theory, Phys. Rev. B 91, 035134 (2015).

[57] C. Wang and M. Levin, Topological invariants for gauge theories and symmetry-protected topological phases, Phys. Rev. B 91, 165119 (2015).

[58] P. Putrov, J. Wang, and S.-T. Yau, Braiding statistics and link invariants of bosonic/fermionic topological quantum matter in $2+1$ and $3+1$ dimensions, Ann. Phys. (Amsterdam) 384, 254 (2017).

[59] J. Wang, X.-G. Wen, and S.-T. Yau, Quantum statistics and spacetime surgery, Phys. Lett. B 807, 135516 (2020).

[60] J. Wang, X.-G. Wen, and S.-T. Yau, Quantum statistics and spacetime topology: Quantum surgery formulas, Ann. Phys. (Amsterdam) 409, 167904 (2019).

[61] A. Prakash, J. Wang, and T.-C. Wei, Unwinding shortrange entanglement, Phys. Rev. B 98, 125108 (2018).

[62] A. Prakash and J. Wang, Unwinding fermionic SPT phases: Supersymmetry extension, Phys. Rev. B 103, 085130 (2021).

[63] Y. Tachikawa, On gauging finite subgroups, SciPost Phys. 8, 015 (2020).

[64] J. Wang, K. Ohmori, P. Putrov, Y. Zheng, Z. Wan, M. Guo, H. Lin, P. Gao, and S.-T. Yau, Tunneling topological vacua via extended operators: (Spin-)TQFT spectra and boundary deconfinement in various dimensions, Prog. Theor. Exp. Phys. 2018, 053A01 (2018).

[65] Z. Wan and J. Wang, Adjoint QCD $_{4}$, Deconfined critical phenomena, symmetry-enriched topological quantum field theory, and higher symmetry-extension, Phys. Rev. D 99, 065013 (2019).

[66] J. McNamara and C. Vafa, Cobordism Classes and the swampland, arXiv:1909.10355.

[67] N. Seiberg, Thoughts About Quantum Field Theory (Talk at Strings 2019) (2019).

[68] L. M. Krauss and F. Wilczek, Discrete Gauge Symmetry in Continuum Theories, Phys. Rev. Lett. 62, 1221 (1989). 
[69] R. Dijkgraaf and E. Witten, Topological gauge theories and group cohomology, Commun. Math. Phys. 129, 393 (1990).

[70] S. Weinberg, A New Light Boson?, Phys. Rev. Lett. 40, 223 (1978).

[71] F. Wilczek, Problem of Strong $P$ and $T$ Invariance in the Presence of Instantons, Phys. Rev. Lett. 40, 279 (1978).

[72] D. Gaiotto, A. Kapustin, Z. Komargodski, and N. Seiberg, Theta, time reversal, and temperature, J. High Energy Phys. 05 (2017) 091.

[73] Z. Wan, J. Wang, and Y. Zheng, Quantum 4d Yang-Mills theory and time-reversal symmetric $5 \mathrm{~d}$ higher-gauge topological field theory, Phys. Rev. D 100, 085012 (2019).

[74] A. Belavin, A. M. Polyakov, A. Schwartz, and Y. Tyupkin, Pseudoparticle solutions of the Yang-Mills equations, Phys. Lett. 59B, 85 (1975).

[75] G. 't Hooft, Computation of the quantum effects due to a four-dimensional pseudoparticle, Phys. Rev. D 14, 3432 (1976).

[76] F. R. Klinkhamer and N. Manton, A saddle point solution in the Weinberg-Salam theory, Phys. Rev. D 30, 2212 (1984).

[77] C. N. Yang and R. L. Mills, Conservation of isotopic spin and isotopic gauge invariance, Phys. Rev. 96, 191 (1954).

[78] T. Banks and N. Seiberg, Symmetries and strings in field theory and gravity, Phys. Rev. D 83, 084019 (2011).

[79] E. Witten, Fermion Path Integrals And Topological Phases, Rev. Mod. Phys. 88, 035001 (2016)..

[80] M. Guo, P. Putrov, and J. Wang, Time reversal, SU(N) Yang-Mills and cobordisms: Interacting topological superconductors/insulators and quantum spin liquids in $3+1$ D, Ann. Phys. 394, 244 (2018).

[81] E. Witten, The "Parity" anomaly on an unorientable manifold, Phys. Rev. B 94, 195150 (2016).

[82] P. Putrov, R. Thorngren, Z. Wan, and J. Wang (to be published).

[83] C. Cordova and K. Ohmori, Anomaly constraints on gapped phases with discrete chiral symmetry, Phys. Rev. D 102, 025011 (2020).

[84] L. Fidkowski and A. Kitaev, Topological phases of fermions in one dimension, Phys. Rev. B 83, 075103 (2011).

[85] L. Fidkowski and A. Kitaev, Effects of interactions on the topological classification of free fermion systems, Phys. Rev. B 81, 134509 (2010).

[86] (to be published).

[87] M. M. Anber and E. Poppitz, Two-flavor adjoint QCD, Phys. Rev. D 98, 034026 (2018).

[88] C. Cordova and T. T. Dumitrescu, Candidate phases for $\mathrm{SU}(2)$ adjoint $\mathrm{QCD}_{4}$ with two flavors from $\mathcal{N}=2$ supersymmetric Yang-Mills theory, arXiv:1806.09592.

[89] Z. Bi and T. Senthil, Adventure in Topological Phase Transitions in $3+1$-D: Non-Abelian Deconfined
Quantum Criticalities and a Possible Duality, Phys. Rev. X 9, 021034 (2019).

[90] J. Wang, Y.-Z. You, and Y. Zheng, Gauge enhanced quantum criticality and time reversal deconfined domain wall: SU(2) Yang-Mills dynamics with topological terms, Phys. Rev. Research 2, 013189 (2020).

[91] H. B. Nielsen and M. Ninomiya, A no-go theorem for regularizing chiral fermions, Phys. Lett. B 105, 219 (1981).

[92] X.-G. Wen, A lattice non-perturbative definition of an SO(10) chiral gauge theory and its induced standard model, Chin. Phys. Lett. 30, 111101 (2013).

[93] J. Wang and X.-G. Wen, Non-perturbative regularization of $1+1 \mathrm{D}$ anomaly-free chiral fermions and bosons: On the equivalence of anomaly matching conditions and boundary gapping rules, arXiv:1307.7480.

[94] Y. You, Y. BenTov, and C. Xu, Interacting topological superconductors and possible origin of $16 n$ chiral fermions in the standard model, arXiv:1402.4151.

[95] Y.-Z. You and C. Xu, Interacting topological insulator and emergent grand unified theory, Phys. Rev. B 91, 125147 (2015).

[96] Y. BenTov and A. Zee, Origin of families and $S O(18)$ grand unification, Phys. Rev. D 93, 065036 (2016).

[97] Y. Kikukawa, Why is the mission impossible?Decoupling the mirror Ginsparg-Wilson fermions in the lattice models for two-dimensional abelian chiral gauge theories, Prog. Theor. Exp. Phys. 2019, 073 B02 (2019).

[98] Y. Kikukawa, On the gauge invariant path-integral measure for the overlap Weyl fermions in 16 of $\mathrm{SO}(10)$, Prog. Theor. Exp. Phys. 2019, 113B03 (2019).

[99] J. Wang and X.-G. Wen, A solution to the 1+1D gauged chiral fermion problem, Phys. Rev. D 99, 111501 (2019).

[100] S. S. Razamat and D. Tong, Gapped chiral fermions, arXiv: 2009.05037.

[101] X.-G. Wen, Classifying gauge anomalies through symmetry-protected trivial orders and classifying gravitational anomalies through topological orders, Phys. Rev. D 88, 045013 (2013).

[102] S. Catterall, Chiral lattice theories from staggered fermions, arXiv:2010.02290.

[103] J. Wang, ultra unification, https://www.youtube.com/ watch?v=tyGIGbzQN4k, also harvard university particle physics lunch (November 30, 2020) and harvard university string lunch (December 4, 2020), (Talk at Erwin Schrödinger International Institute for Mathematics and Physics (ESI). Higher Structures and Field Theory. Week 1-Topological Orders and Higher Structures on August 4th, 2020) (2020), https://www.youtube.com/ results?search_query=ultra+unification+juven+wang. 